\title{
General Relation Valid for Electrolytic Systems
}

\author{
Anna Maria Michałowska-Kaczmarczyk ${ }^{1 *}$, Tadeusz Michałowski² \\ ${ }^{1}$ Department of Oncology, The University Hospital in Cracow, Cracow, Poland \\ ${ }^{2}$ Department of Analytical Chemistry, Technical University of Cracow, Cracow, Poland \\ Email: *michanku@o2.pl
}

Received 2 December 2015; accepted 26 December 2015; published 29 December 2015

Copyright (C) 2015 by authors and Scientific Research Publishing Inc.

This work is licensed under the Creative Commons Attribution International License (CC BY).

http://creativecommons.org/licenses/by/4.0/

(c) (i) Open Access

\section{Abstract}

A simple general relation $P=Q+R+1$ between the number $P$ of kinds of species, the number $Q$ of charge and elemental/core balances and the number $R$ of independent equilibrium constants is deduced, and its validity is confirmed for non-redox and redox electrolytic systems, of different degree of complexity.

\section{Keywords}

\section{Electrolytic Systems, Species, Balances, Equilibrium Constants}

\section{Introduction}

The quantitative, thermodynamic description of any electrolytic system requires prior information on 1) the species present in the system considered; 2) the equilibrium constants; 3) the balances. The balances and expressions for equilibrium constants interrelate molar concentrations of the species in the system. To do it, we should necessarily define these terms in an unambiguous manner. This possibility gives the Generalized Approach to Electrolytic Systems (GATES) [1]-[18] which offers the best tool applicable for thermodynamic resolution of electrolytic systems of any degree of complexity.

This paper refers to batch and dynamic, multicomponent and mono- or multiphase closed systems with condensed (liquid, liquid + solid, liquid1 + liquid2, liquid1 + liquid2 + solid) phases, where quasistatic processes proceed under isobaric + isothermal conditions. The species forming solid phase are marked below in bold letters.

An example of a dynamic procedure is the titration, where $\mathrm{V} \mathrm{mL}$ of titrant $(\mathrm{T})$ is added into $\mathrm{V}_{0} \mathrm{~mL}$ of titrand (D). The $\mathrm{D}+\mathrm{T}$ mixture thus formed involves the related species.

The issue raised in this paper concerns formulation of a general relation

\footnotetext{
${ }^{*}$ Corresponding author.
}

How to cite this paper: Michałowska-Kaczmarczyk, A.M. and Michałowski, T. (2015) General Relation Valid for Electrolytic Systems. Journal of Analytical Sciences, Methods and Instrumentation, 5, 74-85. 


$$
\mathrm{P}=\mathrm{Q}+\mathrm{R}+1
$$

between the number $\mathrm{P}$ of kinds of species, the number $\mathrm{Q}$ of charge and elemental/core balances and the number $\mathrm{R}$ of equilibrium constants, referred to redox and non-redox electrolytic systems, of different degree of complexity. As stated below, the terms: species, equilibrium constants and balances are presented in literature in ambiguous manner. These ambiguities can be avoided when the related terms are in accordance with the GATES principles.

\section{Species and Equilibrium Constants}

Within GATES, the species are considered in their natural form, i.e., as hydrates $X_{i}^{z_{i}} \cdot n_{i w}$ in aqueous media $\left(\mathrm{W}=\mathrm{H}_{2} \mathrm{O}\right.$ ), or as $\mathrm{X}_{\mathrm{i}}^{\mathrm{z}_{\mathrm{i}}} \cdot \mathrm{n}_{\mathrm{iA}_{1}} \cdots \mathrm{n}_{\mathrm{iA}_{\mathrm{S}}}$ in mixed-solvent media [12]-[14]; $\mathrm{n}_{\mathrm{iW}} \geq 0$ and $\mathrm{n}_{\mathrm{iA}_{\mathrm{s}}} \geq 0 \quad(\mathrm{~s}=1, \cdots, \mathrm{S})$ are the mean numbers of molecules of particular solvents $\left(W, A_{s}\right.$ ) attached to (or involved in) $X_{i}^{z_{i}}$. Therefore, e.g. the soluble species with different number of water molecules involved, e.g. $\mathrm{AlO}_{2}^{-}$and $\mathrm{Al}(\mathrm{OH})_{4}^{-}, \mathrm{AsO}_{2}^{-}$and As $(\mathrm{OH})_{4}^{-}, \mathrm{BO}_{2}^{-}, \mathrm{H}_{2} \mathrm{BO}_{3}^{-}$and $\mathrm{B}(\mathrm{OH})_{4}^{-}, \mathrm{IO}_{4}^{-}$and $\mathrm{H}_{4} \mathrm{IO}_{6}^{-}$, are considered equivalently. On the same principle, e.g. the precipitates: $\mathbf{F e}(\mathbf{O H})_{3}$ and $\mathbf{F e O O H}$ are considered equivalently. However, one can find in literature [19] a strange species such as $\mathrm{Al}_{6}(\mathrm{OH})_{15}^{3+}$ that can be equivalently written as $\left(\mathrm{Al}(\mathrm{OH})_{3}\right)_{5} \mathrm{Al}^{3+}$, although soluble hydroxo-complex $\mathrm{Al}(\mathrm{OH})_{3}$ is unknown elsewhere in literature-probably for the same reasons as $\mathrm{Fe}(\mathrm{OH})_{3}$; the ephemera/freak $\left(\mathbf{A l}(\mathbf{O H})_{3}\right)_{5} \mathrm{Al}^{3+}$ is not acceptable, although the precipitate $\mathbf{A l}(\mathbf{O H})_{3}$ is known. Omission of the species $\mathrm{Al}_{6}(\mathrm{OH})_{15}^{3+}$ in the set of species is followed by omission of the related equilibrium constant, i.e. $\quad(\mathrm{P}-1)=\mathrm{Q}+(\mathrm{R}-1)+1$ is equivalent to Equation (1).

When collecting the species related to the system considered from the viewpoint of Equation (1), one can choose between the species containing different number of $\mathrm{H}_{2} \mathrm{O}$ molecules involved in the related formula. This choice is made, however, in the context of accessible physico-chemical knowledge concerning the components considered. For boric acid, ref. [19] provides the equilibrium constant $\mathrm{K}_{1}^{\mathrm{H}}$ of the reaction

$\mathrm{H}^{+}+\mathrm{B}(\mathrm{OH})_{4}^{-}=\mathrm{HB}(\mathrm{OH})_{4}$, whereas ref. [20] provides the values of successive dissociation constants $\mathrm{K}_{1}, \mathrm{~K}_{2}$, $\mathrm{K}_{3}$ for the reactions: $\mathrm{H}_{3} \mathrm{BO}_{3}=\mathrm{H}^{+}+\mathrm{H}_{2} \mathrm{BO}_{3}^{-}, \mathrm{H}_{2} \mathrm{BO}_{3}^{-}=\mathrm{H}^{+}+\mathrm{HBO}_{3}^{2-}, \mathrm{HBO}_{3}^{2-}=\mathrm{H}^{+}+\mathrm{BO}_{3}^{3-}$. In the first option, we consider two species $\left(\mathrm{HB}(\mathrm{OH})_{4}, \mathrm{~B}(\mathrm{OH})_{4}^{-}\right)$and one equilibrium constant; in the second option, we have 4 species $\left(\mathrm{H}_{3} \mathrm{BO}_{3}, \mathrm{H}_{2} \mathrm{BO}_{3}^{-}, \mathrm{HBO}_{3}^{2-}, \mathrm{BO}_{3}^{3-}\right)$ and 3 equilibrium constants. So the increase in the number of constituents by 2 is associated with an increase in the number of equilibrium constants by 2 . This means that ( $\mathrm{P}-2)$ $=\mathrm{Q}+(\mathrm{R}-2)+1$ (or $(\mathrm{P}+2)=\mathrm{Q}+(\mathrm{R}+2)+1)$ is equivalent to Equation (1). Factually, $\mathrm{HB}(\mathrm{OH})_{4} \equiv \mathrm{H}_{5} \mathrm{BO}_{4} \equiv$ $\mathrm{H}_{3} \mathrm{BO}_{3}\left(\mathrm{H}_{2} \mathrm{O}\right)$ is equivalent to $\mathrm{H}_{3} \mathrm{BO}_{3}$. The inclusion/omission in considerations of $m$ independent components is associated with inclusion/omission of $\mathrm{m}$ equilibrium constants; it means that $(\mathrm{P} \pm \mathrm{m})=\mathrm{Q}+(\mathrm{R} \pm \mathrm{m})+1 \Leftrightarrow$ Equation (1).

The value of any equilibrium constant formulated on the basis of mass action law depends on the reaction notation applied for this purpose; e.g., for a polyprotic acid $\mathrm{H}_{n} \mathrm{~L}$, forming the species $\mathrm{H}_{\mathrm{j}} \mathrm{L}^{\mathrm{j}-\mathrm{n}}(\mathrm{j}=0, \cdots$, q), equal numbers of dissociation constants $\left(K_{i}, i=1, \cdots, q\right)$ or stability constants of the proto-complexes $\left(K_{i}^{H}, j=1, \cdots, q\right)$ are formulated; the $K_{i}$ and $K_{i}^{H}$ values are interdependent [21] (p. 155). Similarly, the standard potentials $\left(E_{0}\right)$ and solubility products $\left(\mathrm{K}_{\mathrm{sp}}\right)$ may be formulated in different manners, $\mathrm{E}_{01}$ for $\mathrm{BrO}^{-}+2 \mathrm{H}^{+}+2 \mathrm{e}^{-}=\mathrm{Br}^{-}+\mathrm{H}_{2} \mathrm{O}$ and $\mathrm{E}_{02}$ for $\mathrm{BrO}^{-}+\mathrm{H}_{2} \mathrm{O}+2 \mathrm{e}^{-}=\mathrm{Br}^{-}+2 \mathrm{OH}^{-}$[19], or $\mathrm{K}_{\text {sp } 1}$ for $\mathbf{M g}(\mathbf{O H})_{2}=\mathrm{Mg}^{2+}+2 \mathrm{OH}^{-}$and $\mathrm{K}_{\text {sp } 2}$ for $\mathbf{M g}(\mathbf{O H})_{2}$ $+2 \mathrm{H}^{+}=\mathrm{Mg}^{2+}+2 \mathrm{H}_{2} \mathrm{O}[22] ; \mathrm{E}_{02}=\mathrm{E}_{01}-\vartheta \cdot \mathrm{pK}_{\mathrm{W}}(\vartheta=\mathrm{RT} / \mathrm{F} \cdot \ln 10) ; \mathrm{pK}_{\mathrm{sp} 2}=\mathrm{pK}_{\mathrm{sp} 1}-2 \cdot \mathrm{pK}_{\mathrm{W}}$. In the context with known $\mathrm{pK}_{\mathrm{W}}, \mathrm{pK}_{\mathrm{sp} 1}$ and $\mathrm{E}_{01}$ values, $\mathrm{E}_{02}$ and $\mathrm{K}_{\mathrm{sp} 2}$ are not new/independent equilibrium constants. In the latter notation, suggested also in [23], the term solubility "product" is misleading; from the viewpoint of the "Occam razor" principle, the generalizing term "solubility constant" seems to be a better choice, in this case. Moreover, some moderately soluble in aqueous media solid phases, e.g. $\mathbf{I}_{\mathbf{2}}$ [1], dimethylglyoxime [24] [25], 8-hydroxyquinoline [26], are not characterized by solubility products; in these cases, the molar solubility, s [mol/L], at defined temperature can be considered as an equivalent form of solubility constant related to the solid phase. The 
problem of apparent/redundant number of equilibrium constants in tables of physico-chemical data and the resulting problems involved with inconsistency of these constants were indicated in [11] [27].

The set of stability constants for tartrate $\mathrm{CuL}_{\mathrm{i}}^{+2-2 i} \quad(\mathrm{i}=1, \cdots, 4)$ complexes cited in [19] is controversial, owing to steric hindrances in formation of the postulated complexes, and high ionic charge $(-6)$ of the ion at $i=4$; the possible complexes with $\mathrm{HL}^{-}$ions were not taken there into account-as elsewhere, for other Me-ions. Nevertheless, in the lack of other, competitive equilibrium data, the stability constants for $\mathrm{CuL}_{\mathrm{i}}^{+2-2 \mathrm{i}}$ can be used in the related calculations.

Concluding, any kind of complex species in involved with the related equilibrium constant, and inclusion/omission of independent complex(es) in calculations is involved with inclusion/omission of the related equilibrium constant(s); P and R are on opposite sides of equality sign in Equation (1). Inclusion of a species (or a set of species) in the related balances without prior physicochemical information related to its/their equilibrium constant(s) is pointless.

\section{Balances}

Formulating the balances for electrolytic systems is also ambiguous. First, the most natural form of balance for ions is the charge balance

$$
\sum_{j=1}^{J} z_{j} \cdot\left[X_{j}^{z_{j}}\right]=0
$$

expressing the principle of electroneutrality of the solution [21], of frequent use is the proton balance [28], resulting from interpretation of stoichiometric reaction notations in acid-base systems. However, the proton balance is a linear combination of charge and concentration balances [29].

Within GATES, the term "core balance” is also applied; e.g., $\mathrm{SO}_{4}^{2-}$ is a common core within the set of species: $\mathrm{HSO}_{4}^{-}, \mathrm{SO}_{4}^{2-}$ and $\mathrm{FeSO}_{4}$ (complex) in the balance

$$
\left[\mathrm{HSO}_{4}^{-}\right]+\left[\mathrm{SO}_{4}^{2-}\right]+\left[\mathrm{FeSO}_{4}\right]=\mathrm{C}
$$

related to $\mathrm{C} \mathrm{mol} / \mathrm{L} \mathrm{FeSO}_{4}$ solution. Generally, a core is considered as a cluster of elements with defined composition (expressed by chemical formula) and charge [5]. Frequently, the core balance is equivalent to elemental balance; e.g., the balance (3) for the core $\mathrm{SO}_{4}^{2-}$ is tantamount with the balance for $\mathrm{S}$. For the system $\mathrm{FeSO}_{4}(\mathrm{C})$ $+\mathrm{H}_{2} \mathrm{~S}\left(\mathrm{C}_{1}\right)$, where none synproportionation [7] [16] reactions occur, one can write the elemental balance

$$
\left[\mathrm{HSO}_{4}^{-}\right]+\left[\mathrm{SO}_{4}^{2-}\right]+\left[\mathrm{FeSO}_{4}\right]+[\mathrm{FeS}]+\left[\mathrm{H}_{2} \mathrm{~S}\right]+\left[\mathrm{HS}^{-}\right]+\left[\mathrm{S}^{2-}\right]=\mathrm{C}+\mathrm{C}_{1}
$$

where $[\mathbf{F e S}]$ is the concentration of the precipitate $\mathbf{F e S}$; Equation (4) can be presented as the sum of core (for $\mathrm{SO}_{4}^{2-}$ and $\mathrm{S}^{2-}$ ) balances: Equation (3), and $[\mathbf{F e S}]+\left[\mathrm{H}_{2} \mathrm{~S}\right]+\left[\mathrm{HS}^{-}\right]+\left[\mathrm{S}^{2-}\right]=\mathrm{C}_{1}$. For the system $\mathrm{H}_{2} \mathrm{C}_{2} \mathrm{O}_{4}\left(\mathrm{C}_{1}\right)+$ $\mathrm{Na}_{2} \mathrm{CO}_{3}\left(\mathrm{C}_{2}\right)$, the elemental balance for $\mathrm{C}$, i.e.,

$$
2\left(\left[\mathrm{H}_{2} \mathrm{C}_{2} \mathrm{O}_{4}\right]+\left[\mathrm{HC}_{2} \mathrm{O}_{4}^{-}\right]+\left[\mathrm{C}_{2} \mathrm{O}_{4}^{2-}\right]\right)+\left[\mathrm{H}_{2} \mathrm{CO}_{3}\right]+\left[\mathrm{HCO}_{3}^{-}\right]+\left[\mathrm{CO}_{3}^{2-}\right]=2 \mathrm{C}_{1}+\mathrm{C}_{2}
$$

can be presented as the linear combination of core (for $\mathrm{C}_{2} \mathrm{O}_{4}^{2-}$ and $\mathrm{CO}_{3}^{2-}$ ) balances:

$$
\left[\mathrm{H}_{2} \mathrm{C}_{2} \mathrm{O}_{4}\right]+\left[\mathrm{HC}_{2} \mathrm{O}_{4}^{-}\right]+\left[\mathrm{C}_{2} \mathrm{O}_{4}^{2-}\right]=\mathrm{C}_{1} \text {, and }\left[\mathrm{H}_{2} \mathrm{CO}_{3}\right]+\left[\mathrm{HCO}_{3}^{-}\right]+\left[\mathrm{CO}_{3}^{2-}\right]=\mathrm{C}_{2} \text {. }
$$

However, when $\mathrm{V} \mathrm{mL}$ of $\mathrm{C} \mathrm{mol} / \mathrm{L} \mathrm{KMnO}_{4}$ is added into $\mathrm{V}_{0} \mathrm{~mL}$ of $\mathrm{C}_{0} \mathrm{~mol} / \mathrm{L}$ of acidified $\left(\mathrm{H}_{2} \mathrm{SO}_{4}\right)$ solution of $\mathrm{H}_{2} \mathrm{C}_{2} \mathrm{O}_{4}\left(\mathrm{C}_{0}\right)$, the elemental balance for carbon $\mathrm{C}$ (in closed system)

$$
2\left(\left[\mathrm{H}_{2} \mathrm{C}_{2} \mathrm{O}_{4}\right]+\left[\mathrm{HC}_{2} \mathrm{O}_{4}^{-}\right]+\left[\mathrm{C}_{2} \mathrm{O}_{4}^{2-}\right]\right)+\left[\mathrm{H}_{2} \mathrm{CO}_{3}\right]+\left[\mathrm{HCO}_{3}^{-}\right]+\left[\mathrm{CO}_{3}^{2-}\right]=2 \mathrm{C}_{0} \mathrm{~V}_{0} /\left(\mathrm{V}_{0}+\mathrm{V}\right)
$$

cannot be presented as the (combined) sum of core balances for $\mathrm{C}_{2} \mathrm{O}_{4}^{2-}$ and $\mathrm{CO}_{3}^{2-}$ [9].

The balances are usually formulated for aqueous solutions. For beginners, it may seem to be strange that the concentration balances were not applied for hydrogen $(\mathrm{H})$ and oxygen $(\mathrm{O})$, i.e., for the basic elements, introduced mainly by the solvent $\left(\mathrm{H}_{2} \mathrm{O}\right)$ in aqueous media. The latter issue was not known before formulation (2006) 
[30] [31] of the Approach II to Generalized Electron Balance (GEB). The Approach II to GEB results from linear combination $2 \cdot f(\mathrm{O})-f(H)$ of elemental balances: $f(\mathrm{H})$ for $\mathrm{H}$, and $f(\mathrm{O})$ for $\mathrm{O}$. When referred to aqueous media, the balance $2 \cdot f(O)-f(H)$ and any linear combination of $2 \cdot f(O)-f(H)$ with charge balance (1) and/or other elemental/core balances do not involve the mean numbers $n_{i}=n_{i w}$ of coordinating water molecules at the species $\mathrm{X}_{\mathrm{i}}^{\mathrm{z}_{\mathrm{i}}} \cdot \mathrm{n}_{\mathrm{iW}}$; note that even $\mathrm{n}_{\mathrm{iW}}$ for $\mathrm{H}^{+}$in aqueous media is practically unknown [32] [33]. When referred to non-aqueous or mixed-solvent media where at least one amphiprotic co-solvent is involved, after a proper linear combination of $2 \cdot f(O)-f(H)$ with charge and other $\left(\mathrm{Y}_{\mathrm{p}} \neq \mathrm{H}, \mathrm{O}\right)$ elemental/core balances, all coordination numbers $\mathrm{n}_{\mathrm{iA}_{\mathrm{s}}}(\mathrm{s}=1, \ldots, \mathrm{S})$ related to the species $\mathrm{X}_{\mathrm{i}}^{\mathrm{Z}_{\mathrm{i}}} \cdot \mathrm{n}_{\mathrm{iA}_{1}} \cdots \mathrm{n}_{\mathrm{iA}_{\mathrm{S}}}$ are cancelled, see e.g. [12]-[14].

The balance $2 \cdot f(\mathrm{O})-f(\mathrm{H})$, when referred to redox systems of any degree of complexity, is the balance independent on charge and elemental/core balances for $Y_{p} \neq \mathrm{H}$, O, contrary to non-redox systems [34]. The 2.f(O) $f(H)$ is a starting form for GEB related to a redox system [1] [2] [4]-[18]. A proper choice of multipliers for the combined balances gives the simplest form of GEB [11]-[14]. The $2 \cdot f(\mathrm{O})-f(\mathrm{H})$ and any linear combination of $2 \cdot f(\mathrm{O})-f(\mathrm{H})$ with other balances related to a redox system have full properties of GEB. The GEB obtained according to Approach II is equivalent [5] to GEB obtained according to Approach I [35]-[42], considered as a "short" version of GEB, based on the principle of common pool of electrons for elements participating immediately the equilibria in a redox system. The formulation of GEB according to Approach I can be used, if the oxidation numbers for all elements in the species participating redox equlibria are easy to calculate. Prior knowledge of the oxidation numbers is not necessary if GEB is formulated according to Approach II; it is a circumstance of capital importance, particularly when referred to complex organic species of biological origin. In this context, it should be noticed that the oxidation numbers are assumed arbitrarily. It should also be stated that the roles of oxidizers and reducers are not ascribed to particular species within both Approaches (I and II) to GEB, formulated by Michałowski in 1992 and 2006, respectively.

For comparison, earlier (i.e., after 1960s) approaches to dynamic redox systems were based on primitive formulations, with stoichiometric reactions involved. The roles of oxidizers and reducers were ascribed to indicated species, and homogeneous, non-homogeneous and symmetrical reactions were distinguished. Irrespectively of the complexity of the redox system considered, only two pairs $\left\{\left(\mathrm{Ox}_{\mathrm{i}}, \mathrm{Red}_{\mathrm{i}}\right) \mid \mathrm{i}=1,2\right\}$ were involved in two concentration balances. Charge balance and concentration balances for other elements were not formulated. Only two equilibrium constants, namely "formal" potentials related to the $\left(\mathrm{Ox}_{\mathrm{i}}, \operatorname{Red}_{\mathrm{i}}\right)$ pairs were used and applied to formulate the equilibrium constant for the redox reaction notation considered. These approaches were extensively criticized, mainly in [4] [8]. Nonetheless, those approaches are still practiced, also in literature issued recently [43] [44]. Thus, the wide promotion of GATES/GEB, that provides the best possible thermodynamic approach to electrolytic redox systems, is necessary.

\section{Examples of Electrolytic Systems}

\subsection{Non-Redox Systems}

Example 1. For aqueous solution of the mixture $\mathrm{KCl}\left(\mathrm{C}_{1}\right)+\mathrm{NaNO}_{3}\left(\mathrm{C}_{2}\right)+\mathrm{NaCl}\left(\mathrm{C}_{3}\right)+\mathrm{HCl}\left(\mathrm{C}_{4}\right)+\mathrm{KNO}_{3}\left(\mathrm{C}_{5}\right)$ $+\mathrm{HNO}_{3}\left(\mathrm{C}_{6}\right)$ we have: $\mathrm{P}=7\left(\mathrm{H}_{2} \mathrm{O}, \mathrm{H}^{+}, \mathrm{OH}^{-}, \mathrm{K}^{+}, \mathrm{Na}^{+}, \mathrm{Cl}^{-}, \mathrm{NO}_{3}^{-}\right) ; \mathrm{Q}=5\left(\left[\mathrm{~K}^{+}\right]=\mathrm{C}_{1}+\mathrm{C}_{5},\left[\mathrm{Na}^{+}\right]=\mathrm{C}_{2}+\mathrm{C}_{3},\left[\mathrm{Cl}^{-}\right]=\right.$ $\left.\mathrm{C}_{1}+\mathrm{C}_{4},\left[\mathrm{NO}_{3}^{-}\right]=\mathrm{C}_{2}+\mathrm{C}_{5}+\mathrm{C}_{6},\left[\mathrm{H}^{+}\right]-\left[\mathrm{OH}^{-}\right]+\left[\mathrm{K}^{+}\right]+\left[\mathrm{Na}^{+}\right]-\left[\mathrm{Cl}^{-}\right]-\left[\mathrm{NO}_{3}^{-}\right]=0\right) ; \mathrm{R}=1\left(\mathrm{~K}_{\mathrm{W}}\right) ;$ then $7=1$ $+5+1$.

Example 2. For $\mathrm{NaCl}$ (excess) $+\mathrm{H}_{2} \mathrm{O}$ system: $\mathrm{P}=6\left(\mathrm{H}_{2} \mathrm{O}, \mathrm{H}^{+}, \mathrm{OH}^{-}, \mathrm{Na}^{+}, \mathrm{Cl}^{-}, \mathrm{NaCl}_{(\mathrm{s})}\right) ; \mathrm{Q}=3$ (charge, $\mathrm{Na}, \mathrm{Cl}$ ); $\mathrm{R}=2\left(\mathrm{~K}_{\mathrm{W}}, \mathrm{s}\right)$; then $6=3+2+1$.

$\mathrm{NaCl}$ is usually considered as wholly dissociated in diluted aqueous solutions. However, in the saturated solution, not dissociated forms of this salt are admitted, $[\mathrm{NaCl}]>0$, i.e., $\mathrm{NaCl}$ can be considered as a soluble complex [45]; in this case, we have $\mathrm{P}=7, \mathrm{Q}=3$, $\mathrm{R}=3$, i.e. $\quad 7=3+3+1$. The solubility s of this salt is considered as a kind of equilibrium constant; this assumption can be also related to some other solids. However, the $\mathrm{s}$ should not be considered [24]-[26] as the quantity interrelated with the solubility product $K_{s p}$ for $\mathbf{M}_{\mathbf{n}} \mathbf{L}_{\mathbf{u}}$ by the relation $\mathrm{s}^{\mathrm{n}+\mathrm{u}}=\mathrm{n}^{\mathrm{n}} \mathrm{u}^{\mathrm{u}} \cdot \mathrm{K}_{\mathrm{sp}}$, as is usually practiced, e.g. in [45].

Example 3. $\mathbf{C a C O}_{3}+\mathrm{H}_{2} \mathrm{O}$ system: $\mathrm{P}=11\left(\mathrm{H}_{2} \mathrm{O}, \mathrm{H}^{+}, \mathrm{OH}^{-}, \mathrm{Ca}^{2+}, \mathrm{CaOH}^{+}, \mathrm{CaHCO}_{3}^{+}, \mathrm{CaCO}_{3}, \mathbf{C a C O}_{3(\mathrm{~s})}\right.$ $\left.\mathrm{H}_{2} \mathrm{CO}_{3}, \mathrm{HCO}_{3}^{-}, \mathrm{CO}_{3}^{2-}\right) ; \mathrm{Q}=3, \mathrm{R}=7$; then $11=3+7+1$. 
Example 4. AgCl $+\mathrm{H}_{2} \mathrm{O}+\mathrm{NH}_{3}$ system: $\mathrm{P}=16\left(\mathrm{H}_{2} \mathrm{O}, \mathrm{H}^{+}, \mathrm{OH}^{-}, \mathrm{Ag}^{+}, \mathrm{AgOH}, \mathrm{Ag}(\mathrm{OH})_{2}^{-}, \mathrm{Ag}(\mathrm{OH})_{3}^{2-}, \mathrm{AgCl}\right.$, $\left.\mathrm{AgCl}_{2}^{-}, \mathrm{AgCl}_{3}^{2-}, \mathbf{A g C l}_{(\mathrm{s})}, \mathrm{Cl}^{-}, \mathrm{AgNH}_{3}^{+}, \mathrm{Ag}\left(\mathrm{NH}_{3}\right)_{2}^{+}, \mathrm{NH}_{4}^{+}, \mathrm{NH}_{3}\right), \mathrm{Q}=4, \mathrm{R}=11$; then $16=4+11+1$.

Example 5. AgCl $+\mathrm{H}_{2} \mathrm{O}+\mathrm{NH}_{3}$ (excess) system: $\mathrm{P}=15\left(\mathrm{H}_{2} \mathrm{O}, \mathrm{H}^{+}, \mathrm{OH}^{-}, \mathrm{Ag}^{+}, \mathrm{AgOH}, \mathrm{Ag}(\mathrm{OH})_{2}^{-}\right.$, $\left.\mathrm{Ag}(\mathrm{OH})_{3}^{2-}, \mathrm{AgCl}, \mathrm{AgCl}_{2}^{-}, \mathrm{AgCl}_{3}^{2-}, \mathrm{Cl}^{-}, \mathrm{AgNH}_{3}^{+}, \mathrm{Ag}\left(\mathrm{NH}_{3}\right)_{2}^{+}, \mathrm{NH}_{4}^{+}, \mathrm{NH}_{3}\right) ; \mathrm{Q}=4, \mathrm{R}=10$; then $15=4+$ $10+1$.

Example 6. $\mathrm{HgCl}_{2}+\mathrm{H}_{2} \mathrm{O}+\mathrm{KI}: \mathrm{P}=18\left(\mathrm{H}_{2} \mathrm{O}, \mathrm{H}^{+}, \mathrm{OH}^{-}, \mathrm{Hg}^{2+}, \mathrm{HgOH}^{+}, \mathrm{Hg}(\mathrm{OH})_{2}, \mathrm{HgCl}^{+}, \mathrm{HgCl}_{2}, \mathrm{HgCl}_{3}^{-}\right.$, $\left.\mathrm{HgCl}_{4}^{2-}, \mathrm{HgI}^{+}, \mathrm{HgI}_{2}, \mathrm{HgI}_{3}^{-}, \mathrm{HgI}_{4}^{2-}, \mathbf{H g I}_{2(\mathrm{~s})}, \mathrm{I}^{-}, \mathrm{Cl}^{-}, \mathrm{K}^{+}\right), \mathrm{Q}=5, \mathrm{R}=12$; then $18=5+12+1$.

Example 7. $\mathrm{HgCl}_{2}+\mathrm{H}_{2} \mathrm{O}+\mathrm{KI}$ (excess) : $\mathrm{P}=17\left(\mathrm{H}_{2} \mathrm{O}, \mathrm{H}^{+}, \mathrm{OH}^{-}, \mathrm{Hg}^{2+}, \mathrm{HgOH}^{+}, \mathrm{Hg}(\mathrm{OH})_{2}, \mathrm{HgCl}^{+}, \mathrm{HgCl}_{2}\right.$, $\left.\mathrm{HgCl}_{3}^{-}, \mathrm{HgCl}_{4}^{2-}, \mathrm{HgI}^{+}, \mathrm{HgI}_{2}, \mathrm{HgI}_{3}^{-}, \mathrm{HgI}_{4}^{2-}, \mathrm{I}^{-}, \mathrm{Cl}^{-}, \mathrm{K}^{+}\right), \mathrm{Q}=5, \mathrm{R}=11$; then $17=5+11+1$.

Example 8. Struvite (pr1 $=\mathbf{M g N H}_{4} \mathbf{P O}_{4}$ ) is introduced into $\mathrm{V} \mathrm{mL}$ of aqueous solution with dissolved $\mathrm{CO}_{2}$. As results from detailed calculations made in [46], before the solubility product $\left(\mathrm{K}_{\mathrm{sp} 1}\right)$ for pr1 is attained, the solubility product $\left(\mathrm{K}_{\mathrm{sp} 2}\right)$ for pr2 $=\mathbf{M g}_{3}\left(\mathbf{P O}_{4}\right)_{2}$ is crossed. At $\left(\mathrm{pC}_{0}, \mathrm{pC}_{\mathrm{CO} 2}, \mathrm{pC}_{\mathrm{b}}\right)=(3,4, \infty)$, the process pr1 $\rightarrow$ pr2 leads to total depletion of pr1; the solubility product $\mathrm{K}_{\mathrm{sp} 1}$ is not attained $\left(\mathrm{q}_{1}<1\right)$. At $\left(\mathrm{pC}_{0}, \mathrm{pC}_{\mathrm{CO} 2}, \mathrm{pC}_{\mathrm{b}}\right)=(2,4, \infty)$, $\mathrm{K}_{\mathrm{sp} 2}$ for pr2 is attained at ppr1 $=2.013$ and pr2 precipitates according to reaction 3pr1 $=\mathrm{pr} 2+\mathrm{HPO}_{4}^{2-}+$ $2 \mathrm{NH}_{4}^{+}+\mathrm{NH}_{3}$ up to ppr1 $=2.362$, where the solubility product for pr 1 is crossed and the dissolution process pr 1 $\rightarrow$ pr2 is terminated. At equilibrium, the solid phase consists of the two species: pr1 and pr2, and the expressions for $\mathrm{K}_{\mathrm{sp} 1}$ and $\mathrm{K}_{\mathrm{sp} 2}$ are valid. Then at $\left(\mathrm{pC}_{0}, \mathrm{pC}_{\mathrm{CO} 2}, \mathrm{pC}_{\mathrm{b}}\right)=(3,4, \infty)$ we have: $\mathrm{P}=23, \mathrm{Q}=5, \mathrm{R}=17$; then $23=17+$ $5+1$. At $\left(\mathrm{pC}_{0}, \mathrm{pC}_{\mathrm{CO} 2}, \mathrm{pC}_{\mathrm{b}}\right)=(2,4, \infty)$ we have: $\mathrm{P}=24, \mathrm{Q}=5, \mathrm{R}=18$; then $24=18+5+1$.

Example 9. For the Liebig-Denigès titration, described in [47], where particular species are presented, we have: a) at the step before precipitation of AgI: $\mathrm{P}=24, \mathrm{Q}=8, \mathrm{R}=15$; then $24=8+15+1$; b) at the step after AgI precipitation: $\mathrm{P}=25, \mathrm{Q}=8$ and $\mathrm{R}=16$; then $25=8+16+1$, see also [34].

Example 10. $\mathrm{V}_{0} \mathrm{~mL}$ of $\mathrm{ZnSO}_{4}\left(\mathrm{C}_{0}\right)+\mathrm{NH}_{3}\left(\mathrm{C}_{01}\right)+\mathrm{NH}_{4} \mathrm{Cl}\left(\mathrm{C}_{02}\right)+\mathrm{NaH}_{2} \mathrm{In}=\mathrm{C}_{20} \mathrm{H}_{12} \mathrm{~N}_{3} \mathrm{O}_{7} \mathrm{SNa}$ (erio T) solution is titrated with $\mathrm{V} \mathrm{mL}$ of $\mathrm{C} \mathrm{mol} / \mathrm{L}$ EDTA $=\mathrm{C}_{10} \mathrm{H}_{14} \mathrm{~N}_{2} \mathrm{O}_{8} \mathrm{Na}_{2}$. On the basis of the data presented in [34] [37], we have: $\mathrm{P}=36, \mathrm{Q}=8, \mathrm{R}=27$, and then $36=8+27+1$, see also [1].

\subsection{Redox Systems}

Example 11. For aqueous solution of $\mathrm{C} \mathrm{mol} / \mathrm{L} \mathrm{Br}_{2}$ we have [35] [36]: $\mathrm{P}=11\left(\mathrm{H}_{2} \mathrm{O}, \mathrm{H}^{+}, \mathrm{OH}^{-}, \mathrm{e}^{-}, \mathrm{HBrO}_{3}\right.$, $\left.\mathrm{BrO}_{3}^{-}, \mathrm{HBrO}, \mathrm{BrO}^{-}, \mathrm{Br}_{2}, \mathrm{Br}_{3}^{-}, \mathrm{Br}^{-}\right) ; \mathrm{Q}=3\left((\mathrm{Z}-5)\left(\left[\mathrm{HBrO}_{3}\right]+\left[\mathrm{BrO}_{3}^{-}\right]\right)+(\mathrm{Z}-1)\left([\mathrm{HBrO}]+\left[\mathrm{BrO}^{-}\right]\right)+2 \mathrm{Z}\left[\mathrm{Br}_{2}\right]+\right.$ $(\mathrm{Z}+1)\left[\mathrm{Br}^{-}\right]=2 \mathrm{ZC}$, where $\mathrm{Z}=35$-atomic number for $\left.\mathrm{Br} ; \mathrm{H}^{+}\right]-\left[\mathrm{OH}^{-}\right]-\left[\mathrm{BrO}_{3}^{-}\right]-\left[\mathrm{BrO}^{-}\right]-\left[\mathrm{Br}_{3}^{-}\right]-\left[\mathrm{Br}^{-}\right]=$ $\left.0 ;\left[\mathrm{HBrO}_{3}\right]+\left[\mathrm{BrO}_{3}^{-}\right]+[\mathrm{HBrO}]+\left[\mathrm{BrO}^{-}\right]+2\left[\mathrm{Br}_{2}\right]+3\left[\mathrm{Br}_{3}^{-}\right]+\left[\mathrm{Br}^{-}\right]=2 \mathrm{C}\right) ; \mathrm{R}=7$

$\left(\mathrm{K}_{\mathrm{W}}, 10^{0.7} \cdot\left[\mathrm{H}^{+}\right]\left[\mathrm{BrO}_{3}^{-}\right]=\left[\mathrm{HBrO}_{3}\right], 10^{8.6} \cdot\left[\mathrm{H}^{+}\right]\left[\mathrm{BrO}^{-}\right]=[\mathrm{HBrO}],\left[\mathrm{BrO}_{3}^{-}\right]=\left[\mathrm{Br}^{-}\right] \cdot 10^{6 \mathrm{~A}(\mathrm{E}-1.45)+6 \mathrm{pH}},\left[\mathrm{BrO}^{-}\right]=\right.$ $\left[\mathrm{Br}^{-}\right] \cdot 10^{2 \mathrm{~A}(\mathrm{E}-0.76)+2 \mathrm{pH}-28},\left[\mathrm{Br}_{2}\right]=\left[\mathrm{Br}^{-}\right]^{2} \cdot 10^{2 \mathrm{~A}(\mathrm{E}-1.087)},\left[\mathrm{Br}_{3}^{-}\right]=\left[\mathrm{Br}^{-}\right]^{3} \cdot 10^{2 \mathrm{~A}(\mathrm{E}-1.05)}$, where $\mathrm{A}=16.92$ at $25^{\circ} \mathrm{C}$; then $11=3+7+1$.

Example 11a. For $\mathrm{C} \mathrm{mol} / \mathrm{L} \mathrm{NaBrO}$, we have $12=4+7+1$.

Example 12. $\mathrm{V} \mathrm{mL}$ of $\mathrm{KMnO}_{4}(\mathrm{C})+\mathrm{CO}_{2}\left(\mathrm{C}_{1}\right)$ as $\mathrm{T}$ is added into $\mathrm{V}_{0} \mathrm{~mL}$ of $\mathrm{D} \mathrm{FeSO}_{4}\left(\mathrm{C}_{01}\right)+\mathrm{H}_{2} \mathrm{C}_{2} \mathrm{O}_{4}\left(\mathrm{C}_{02}\right)+$ $\mathrm{H}_{2} \mathrm{SO}_{4}\left(\mathrm{C}_{03}\right)+\mathrm{CO}_{2}\left(\mathrm{C}_{04}\right)$. This system involves $\mathrm{P}=39$ species: $\mathrm{H}_{2} \mathrm{O}, \mathrm{H}^{+}, \mathrm{OH}^{-}, \mathrm{e}^{-}, \mathrm{K}^{+}, \mathrm{HSO}_{4}^{-}, \mathrm{SO}_{4}^{2-}, \mathrm{H}_{2} \mathrm{C}_{2} \mathrm{O}_{4}$, $\mathrm{HC}_{2} \mathrm{O}_{4}^{-}, \mathrm{C}_{2} \mathrm{O}_{4}^{2-}, \mathrm{H}_{2} \mathrm{CO}_{3}, \mathrm{HCO}_{3}^{-}, \mathrm{CO}_{3}^{2-}, \mathrm{MnO}_{4}^{-}, \mathrm{MnO}_{4}^{2-}, \mathrm{Mn}^{3+}, \mathrm{MnOH}^{2+}, \mathrm{MnC}_{2} \mathrm{O}_{4}^{+}, \mathrm{Mn}\left(\mathrm{C}_{2} \mathrm{O}_{4}\right)_{2}^{-}$, $\mathrm{Mn}\left(\mathrm{C}_{2} \mathrm{O}_{4}\right)_{3}^{3-}, \mathrm{Mn}^{2+}, \mathrm{MnOH}^{+}, \mathrm{MnSO}_{4}, \mathrm{MnC}_{2} \mathrm{O}_{4}, \mathrm{Mn}\left(\mathrm{C}_{2} \mathrm{O}_{4}\right)_{2}^{2-}, \mathrm{Fe}^{2+}, \mathrm{FeOH}^{+}, \mathrm{FeSO}_{4}, \mathrm{Fe}\left(\mathrm{C}_{2} \mathrm{O}_{4}\right)_{2}^{2-}, \mathrm{Fe}\left(\mathrm{C}_{2} \mathrm{O}_{4}\right)_{3}^{4-}$, $\mathrm{Fe}^{3+}, \mathrm{FeOH}^{2+}, \mathrm{Fe}(\mathrm{OH})_{2}^{+}, \mathrm{Fe}_{2}(\mathrm{OH})_{2}^{4+}, \mathrm{FeSO}_{4}^{+}, \mathrm{Fe}\left(\mathrm{SO}_{4}\right)_{2}^{-}, \quad \mathrm{FeC}_{2} \mathrm{O}_{4}^{+}, \mathrm{Fe}\left(\mathrm{C}_{2} \mathrm{O}_{4}\right)_{2}^{-}, \quad \mathrm{Fe}\left(\mathrm{C}_{2} \mathrm{O}_{4}\right)_{3}^{3-}$ under assumption that $\mathrm{C}_{03}$ value is sufficiently high to prevent precipitation of $\mathbf{M n O}_{2}, \mathbf{F e C}_{2} \mathbf{O}_{4}$ and $\mathbf{M n C}_{2} \mathbf{O}_{4}$, i.e. the solubility products of these precipitates are not crossed [9].

Three electron-active elements: $\mathrm{Fe}, \mathrm{C}$ and $\mathrm{Mn}$ are involved in this system. Denoting atomic numbers: $\mathrm{Z}_{\mathrm{C}}=6$ for $\mathrm{C}, \mathrm{Z}_{\mathrm{Mn}}=25$ for $\mathrm{Mn}, \mathrm{Z}_{\mathrm{Fe}}=26$ for Fe, the resulting GEB is written according to Approach I to GEB as follows: 


$$
\begin{aligned}
& \left(\mathrm{Z}_{\mathrm{Fe}}-2\right)\left(\left[\mathrm{Fe}^{2+}\right]+\left[\mathrm{FeOH}^{+}\right]+\left[\mathrm{FeSO}_{4}\right]\right)+\left(\mathrm{Z}_{\mathrm{Fe}}-3\right)\left(\left[\mathrm{Fe}^{3+}\right]+\left[\mathrm{FeOH}^{2+}\right]+\left[\mathrm{Fe}(\mathrm{OH})_{2}^{+}\right]+2\left[\mathrm{Fe}_{2}(\mathrm{OH})_{2}^{4+}\right]\right. \\
& \left.+\left[\mathrm{FeSO}_{4}^{+}\right]+\left[\mathrm{Fe}\left(\mathrm{SO}_{4}\right)_{2}^{-}\right]\right)+\left(\mathrm{Z}_{\mathrm{Fe}}-2+4 \cdot\left(\mathrm{Z}_{\mathrm{C}}-3\right)\right)\left[\mathrm{Fe}\left(\mathrm{C}_{2} \mathrm{O}_{4}\right)_{2}^{2-}\right]+\left(\mathrm{Z}_{\mathrm{Fe}}-2+6 \cdot\left(\mathrm{Z}_{\mathrm{C}}-3\right)\right)\left[\mathrm{Fe}\left(\mathrm{C}_{2} \mathrm{O}_{4}\right)_{3}^{4-}\right] \\
& +\left(\mathrm{Z}_{\mathrm{Fe}}-3+2 \cdot\left(\mathrm{Z}_{\mathrm{C}}-3\right)\right)\left[\mathrm{FeC}_{2} \mathrm{O}_{4}^{+}\right]+\left(\mathrm{Z}_{\mathrm{Fe}}-3+4 \cdot\left(\mathrm{Z}_{\mathrm{C}}-3\right)\right)\left[\mathrm{Fe}\left(\mathrm{C}_{2} \mathrm{O}_{4}\right)_{2}^{-}\right] \\
& +\left(\mathrm{Z}_{\mathrm{Fe}}-3+6 \cdot\left(\mathrm{Z}_{\mathrm{C}}-3\right)\right)\left[\mathrm{Fe}\left(\mathrm{C}_{2} \mathrm{O}_{4}\right)_{3}^{3-}\right]+2\left(\mathrm{Z}_{\mathrm{C}}-3\right)\left(\left[\mathrm{H}_{2} \mathrm{C}_{2} \mathrm{O}_{3}\right]+\left[\mathrm{HC}_{2} \mathrm{O}_{3}^{-}\right]+\left[\mathrm{C}_{2} \mathrm{O}_{3}^{2-}\right]\right) \\
& +\left(\mathrm{Z}_{\mathrm{C}}-4\right)\left(\left[\mathrm{H}_{2} \mathrm{CO}_{3}\right]+\left[\mathrm{HCO}_{3}^{-}\right]+\left[\mathrm{CO}_{3}^{2-}\right]\right)+\left(\mathrm{Z}_{\mathrm{Mn}}-7\right)\left[\mathrm{MnO}_{4}^{-}\right]+\left(\mathrm{Z}_{\mathrm{Mn}}-6\right)\left[\mathrm{MnO}_{4}^{2-}\right] \\
& +\left(\mathrm{Z}_{\mathrm{Mn}}-3\right)\left(\left[\mathrm{Mn}^{3+}\right]+\left[\mathrm{MnOH}^{2+}\right]\right)+\left(\mathrm{Z}_{\mathrm{Mn}}-3+2 \cdot\left(\mathrm{Z}_{\mathrm{C}}-3\right)\right)\left[\mathrm{MnC}_{2} \mathrm{O}_{4}^{+}\right] \\
& +\left(\mathrm{Z}_{\mathrm{Mn}}-3+4 \cdot\left(\mathrm{Z}_{\mathrm{C}}-3\right)\right)\left[\mathrm{Mn}\left(\mathrm{C}_{2} \mathrm{O}_{4}\right)_{2}^{-}\right]+\left(\mathrm{Z}_{\mathrm{Mn}}-3+6 \cdot\left(\mathrm{Z}_{\mathrm{C}}-3\right)\right)\left[\mathrm{Mn}\left(\mathrm{C}_{2} \mathrm{O}_{4}\right)_{3}^{3-}\right] \\
& +\left(\mathrm{Z}_{\mathrm{Mn}}-2\right)\left(\left[\mathrm{Mn}^{2+}\right]+\left[\mathrm{MnOH}^{+}\right]+\left[\mathrm{MnSO}_{4}\right]\right)+\left(\mathrm{Z}_{\mathrm{Mn}}-2+2 \cdot\left(\mathrm{Z}_{\mathrm{C}}-3\right)\right)\left[\mathrm{MnC}_{2} \mathrm{O}_{4}\right] \\
& +\left(\mathrm{Z}_{\mathrm{Mn}}-2+4 \cdot\left(\mathrm{Z}_{\mathrm{C}}-3\right)\right)\left[\mathrm{Mn}\left(\mathrm{C}_{2} \mathrm{O}_{4}\right)_{2}^{2-}\right] \\
& =\left(\left(\mathrm{Z}_{\mathrm{Fe}}-2\right) \mathrm{C}_{01} \mathrm{~V}_{0}+2 \cdot\left(\mathrm{Z}_{\mathrm{C}}-3\right) \mathrm{C}_{02} \mathrm{~V}_{0}+\left(\mathrm{Z}_{\mathrm{C}}-4\right)\left(\mathrm{C}_{04} \mathrm{~V}_{0}+\mathrm{C}_{1} \mathrm{~V}\right)+\left(\mathrm{Z}_{\mathrm{Mn}}-7\right) \mathrm{CV}\right) /\left(\mathrm{V}_{0}+\mathrm{V}\right)
\end{aligned}
$$

The equation for charge balance (Equation (7)) and equations for concentration balances for Fe (Equation (8)), Mn (Equation (9)), C (Equation 10) and $\mathrm{SO}_{4}$ (Equation (11)), and $\mathrm{K}$ (Equation (12)) are as follows:

$$
\begin{aligned}
& {\left[\mathrm{H}^{+}\right]-\left[\mathrm{OH}^{-}\right]+\left[\mathrm{K}^{+}\right]-\left[\mathrm{HSO}_{4}^{-}\right]-2\left[\mathrm{SO}_{4}^{2-}\right]-\left[\mathrm{HC}_{2} \mathrm{O}_{4}^{-}\right]-2\left[\mathrm{C}_{2} \mathrm{O}_{4}^{2-}\right]-\left[\mathrm{MnO}_{4}^{-}\right]} \\
& -2\left[\mathrm{MnO}_{4}^{2-}\right]+3\left[\mathrm{Mn}^{3+}\right]+2\left[\mathrm{MnOH}^{2+}\right]+\left[\mathrm{MnC}_{2} \mathrm{O}_{4}^{+}\right]-\left[\mathrm{Mn}\left(\mathrm{C}_{2} \mathrm{O}_{4}\right)_{2}^{-}\right]-3\left[\mathrm{Mn}\left(\mathrm{C}_{2} \mathrm{O}_{4}\right)_{3}^{3-}\right] \\
& +2\left[\mathrm{Mn}^{2+}\right]+\left[\mathrm{MnOH}^{+}\right]-2\left[\mathrm{Mn}\left(\mathrm{C}_{2} \mathrm{O}_{4}\right)_{2}^{2-}\right]+2\left[\mathrm{Fe}^{2+}\right]+\left[\mathrm{FeOH}^{+}\right]-2\left[\mathrm{Fe}\left(\mathrm{C}_{2} \mathrm{O}_{4}\right)_{2}^{2-}\right] \\
& -4\left[\mathrm{Fe}\left(\mathrm{C}_{2} \mathrm{O}_{4}\right)_{3}^{4-}\right]+3\left[\mathrm{Fe}^{3+}\right]+2\left[\mathrm{FeOH}^{2+}\right]+\left[\mathrm{Fe}(\mathrm{OH})_{2}^{+}\right]+4\left[\mathrm{Fe}_{2}(\mathrm{OH})_{2}^{4+}\right] \\
& +\left[\mathrm{FeSO}_{4}^{+}\right]-\left[\mathrm{Fe}\left(\mathrm{SO}_{4}\right)_{2}^{-}\right]+\left[\mathrm{FeC}_{2} \mathrm{O}_{4}^{+}\right]-\left[\mathrm{Fe}\left(\mathrm{C}_{2} \mathrm{O}_{4}\right)_{2}^{-}\right]-3\left[\mathrm{Fe}\left(\mathrm{C}_{2} \mathrm{O}_{4}\right)_{3}^{3-}\right]=0 \\
& {\left[\mathrm{Fe}^{2+}\right]+\left[\mathrm{FeOH}^{+}\right]+\left[\mathrm{FeSO}_{4}\right]+\left[\mathrm{Fe}\left(\mathrm{C}_{2} \mathrm{O}_{4}\right)_{2}^{2-}\right]+\left[\mathrm{Fe}\left(\mathrm{C}_{2} \mathrm{O}_{4}\right)_{3}^{4+}\right]+\left[\mathrm{Fe}^{3+}\right]} \\
& +\left[\mathrm{FeOH}^{2+}\right]+\left[\mathrm{Fe}(\mathrm{OH})_{2}^{+}\right]+2\left[\mathrm{Fe}_{2}(\mathrm{OH})_{2}^{4+}\right]+\left[\mathrm{FeSO}_{4}^{+}\right]+\left[\mathrm{Fe}\left(\mathrm{SO}_{4}\right)_{2}^{-}\right] \\
& +\left[\mathrm{FeC}_{2} \mathrm{O}_{4}^{+}\right]+\left[\mathrm{Fe}\left(\mathrm{C}_{2} \mathrm{O}_{4}\right)_{2}^{-}\right]+\left[\mathrm{Fe}\left(\mathrm{C}_{2} \mathrm{O}_{4}\right)_{3}^{3-}\right]=\mathrm{C}_{01} \mathrm{~V}_{0} /\left(\mathrm{V}_{0}+\mathrm{V}\right) \\
& {\left[\mathrm{MnO}_{4}^{-}\right]+\left[\mathrm{MnO}_{4}^{2-}\right]+\left[\mathrm{Mn}^{3+}\right]+\left[\mathrm{MnOH}^{2+}\right]+\left[\mathrm{MnC}_{2} \mathrm{O}_{4}^{+}\right]+\left[\mathrm{Mn}\left(\mathrm{C}_{2} \mathrm{O}_{4}\right)_{2}^{-}\right]+\left[\mathrm{Mn}\left(\mathrm{C}_{2} \mathrm{O}_{4}\right)_{3}^{3-}\right]} \\
& +\left[\mathrm{Mn}^{2+}\right]+\left[\mathrm{MnOH}^{+}\right]+\left[\mathrm{MnSO}_{4}\right]+\left[\mathrm{MnC}_{2} \mathrm{O}_{4}\right]+\left[\mathrm{Mn}\left(\mathrm{C}_{2} \mathrm{O}_{4}\right)_{2}^{2-}\right]=\mathrm{CV} /\left(\mathrm{V}_{0}+\mathrm{V}\right) \\
& 2\left[\mathrm{H}_{2} \mathrm{C}_{2} \mathrm{O}_{4}\right]+2\left[\mathrm{HC}_{2} \mathrm{O}_{4}^{-}\right]+2\left[\mathrm{C}_{2} \mathrm{O}_{4}^{2-}\right]+\left[\mathrm{H}_{2} \mathrm{CO}_{3}\right]+\left[\mathrm{HCO}_{3}^{-}\right]+\left[\mathrm{CO}_{3}^{2-}\right] \\
& +2 \cdot\left[\mathrm{MnC}_{2} \mathrm{O}_{4}^{+}\right]+4 \cdot\left[\mathrm{Mn}\left(\mathrm{C}_{2} \mathrm{O}_{4}\right)_{2}^{-}\right]+6 \cdot\left[\mathrm{Mn}\left(\mathrm{C}_{2} \mathrm{O}_{4}\right)_{3}^{3-}\right]+2 \cdot\left[\mathrm{MnC}_{2} \mathrm{O}_{4}\right] \\
& +4 \cdot\left[\mathrm{Mn}\left(\mathrm{C}_{2} \mathrm{O}_{4}\right)_{2}^{2-}\right]+4 \cdot\left[\mathrm{Fe}\left(\mathrm{C}_{2} \mathrm{O}_{4}\right)_{2}^{2-}\right]+6 \cdot\left[\mathrm{Fe}\left(\mathrm{C}_{2} \mathrm{O}_{4}\right)_{3}^{4-}\right]+2 \cdot\left[\mathrm{FeC}_{2} \mathrm{O}_{4}^{+}\right] \\
& +4 \cdot\left[\mathrm{Fe}\left(\mathrm{C}_{2} \mathrm{O}_{4}\right)_{2}^{-}\right]+6 \cdot\left[\mathrm{Fe}\left(\mathrm{C}_{2} \mathrm{O}_{4}\right)_{3}^{3-}\right]=\left(2 \mathrm{C}_{02} \mathrm{~V}_{0}+\mathrm{C}_{04} \mathrm{~V}_{0}+\mathrm{C}_{1} \mathrm{~V}\right) /\left(\mathrm{V}_{0}+\mathrm{V}\right) \\
& {\left[\mathrm{HSO}_{4}^{-}\right]+\left[\mathrm{SO}_{4}^{2-}\right]+\left[\mathrm{MnSO}_{4}\right]+\left[\mathrm{FeSO}_{4}\right]+\left[\mathrm{FeSO}_{4}^{+}\right]+2\left[\mathrm{Fe}\left(\mathrm{SO}_{4}\right)_{2}^{-}\right]=\left(\mathrm{C}_{01} \mathrm{~V}_{0}+\mathrm{C}_{03} \mathrm{~V}_{0}\right) /\left(\mathrm{V}_{0}+\mathrm{V}\right)} \\
& {\left[\mathrm{K}^{+}\right]=\mathrm{CV} /\left(\mathrm{V}_{0}+\mathrm{V}\right)}
\end{aligned}
$$

The relationships between concentrations of the species in Equations (6)-(12) are formulated on the basis of 
equilibrium constants, involved in $\mathrm{R}=31$ relations for: $\mathrm{K}_{\mathrm{W}}$ and:

$$
\begin{aligned}
& {\left[\mathrm{H}_{2} \mathrm{C}_{2} \mathrm{O}_{4}\right]=10^{5.2} \cdot\left[\mathrm{H}^{+}\right]^{2}\left[\mathrm{C}_{2} \mathrm{O}_{4}^{2-}\right],\left[\mathrm{HC}_{2} \mathrm{O}_{4}^{-}\right]=10^{3.8} \cdot\left[\mathrm{H}^{+}\right]\left[\mathrm{C}_{2} \mathrm{O}_{4}^{2-}\right] \text {, }} \\
& {\left[\mathrm{H}_{2} \mathrm{CO}_{3}\right]=10^{16.4} \cdot\left[\mathrm{H}^{+}\right]^{2}\left[\mathrm{CO}_{3}^{2-}\right],\left[\mathrm{HCO}_{3}^{-}\right]=10^{10.1} \cdot\left[\mathrm{H}^{+}\right]\left[\mathrm{CO}_{3}^{2-}\right] \text {, }} \\
& {\left[\mathrm{HSO}_{4}^{-}\right]=10^{1.8} \cdot\left[\mathrm{H}^{+}\right]\left[\mathrm{SO}_{4}^{2-}\right],\left[\mathrm{FeOH}^{+}\right]=10^{4.5} \cdot\left[\mathrm{Fe}^{2+}\right]\left[\mathrm{OH}^{-}\right] \text {, }} \\
& {\left[\mathrm{FeOH}^{2+}\right]=10^{11.0} \cdot\left[\mathrm{Fe}^{3+}\right]\left[\mathrm{OH}^{-}\right],\left[\mathrm{Fe}(\mathrm{OH})_{2}^{+}\right]=10^{21.7} \cdot\left[\mathrm{Fe}^{3+}\right]\left[\mathrm{OH}^{-}\right]^{2} \text {, }} \\
& {\left[\mathrm{Fe}_{2}(\mathrm{OH})_{2}^{4+}\right]=10^{25.1} \cdot\left[\mathrm{Fe}^{3+}\right]^{2}\left[\mathrm{OH}^{-}\right]^{2},\left[\mathrm{MnOH}^{+}\right]=10^{3.4} \cdot\left[\mathrm{Mn}^{2+}\right]\left[\mathrm{OH}^{-}\right] \text {, }} \\
& {\left[\mathrm{FeSO}_{4}\right]=10^{2.3} \cdot\left[\mathrm{Fe}^{2+}\right]\left[\mathrm{SO}_{4}^{2-}\right],\left[\mathrm{FeSO}_{4}^{+}\right]=10^{4.18} \cdot\left[\mathrm{Fe}^{3+}\right]\left[\mathrm{SO}_{4}^{2-}\right],} \\
& {\left[\mathrm{Fe}\left(\mathrm{SO}_{4}\right)_{2}^{-}\right]=10^{7.4} \cdot\left[\mathrm{Fe}^{3+}\right]\left[\mathrm{SO}_{4}^{2-}\right]^{2},\left[\mathrm{MnSO}_{4}\right]=10^{2.28} \cdot\left[\mathrm{Mn}^{2+}\right]\left[\mathrm{SO}_{4}^{2-}\right] \text {, }} \\
& {\left[\mathrm{Fe}\left(\mathrm{C}_{2} \mathrm{O}_{4}\right)_{2}^{2-}\right]=10^{4.52} \cdot\left[\mathrm{Fe}^{2+}\right]\left[\mathrm{C}_{2} \mathrm{O}_{4}^{2-}\right]^{2},\left[\mathrm{Fe}\left(\mathrm{C}_{2} \mathrm{O}_{4}\right)_{3}^{4-}\right]=10^{5.22} \cdot\left[\mathrm{Fe}^{2+}\right]\left[\mathrm{C}_{2} \mathrm{O}_{4}^{2-}\right]^{3} \text {, }} \\
& {\left[\mathrm{FeC}_{2} \mathrm{O}_{4}^{+}\right]=10^{7.53} \cdot\left[\mathrm{Fe}^{3+}\right]\left[\mathrm{C}_{2} \mathrm{O}_{4}^{2-}\right],\left[\mathrm{Fe}\left(\mathrm{C}_{2} \mathrm{O}_{4}\right)_{2}^{-}\right]=10^{13.64} \cdot\left[\mathrm{Fe}^{3+}\right]\left[\mathrm{C}_{2} \mathrm{O}_{4}^{2-}\right]^{2},} \\
& {\left[\mathrm{Fe}\left(\mathrm{C}_{2} \mathrm{O}_{4}\right)_{3}^{3-}\right]=10^{18.49} \cdot\left[\mathrm{Fe}^{3+}\right]\left[\mathrm{C}_{2} \mathrm{O}_{4}^{2-}\right]^{3} ;\left[\mathrm{MnC}_{2} \mathrm{O}_{4}\right]=10^{3.82} \cdot\left[\mathrm{Mn}^{2+}\right]\left[\mathrm{C}_{2} \mathrm{O}_{4}^{2-}\right] \text {; }} \\
& {\left[\mathrm{Mn}\left(\mathrm{C}_{2} \mathrm{O}_{4}\right)_{2}^{2-}\right]=10^{5.25} \cdot\left[\mathrm{Mn}^{2+}\right]\left[\mathrm{C}_{2} \mathrm{O}_{4}^{2-}\right]^{2},\left[\mathrm{MnC}_{2} \mathrm{O}_{4}^{+}\right]=10^{9.98} \cdot\left[\mathrm{Mn}^{3+}\right]\left[\mathrm{C}_{2} \mathrm{O}_{4}^{2-}\right] \text {, }} \\
& {\left[\mathrm{Mn}\left(\mathrm{C}_{2} \mathrm{O}_{4}\right)_{2}^{-}\right]=10^{16.57} \cdot\left[\mathrm{Mn}^{3+}\right]\left[\mathrm{C}_{2} \mathrm{O}_{4}^{2-}\right]^{2},\left[\mathrm{Mn}\left(\mathrm{C}_{2} \mathrm{O}_{4}\right)_{3}^{3-}\right]=10^{19.42} \cdot\left[\mathrm{Mn}^{3+}\right]\left[\mathrm{C}_{2} \mathrm{O}_{4}^{2-}\right]^{3} \text {, }} \\
& {\left[\mathrm{MnO}_{4}^{-}\right]=\left[\mathrm{Mn}^{2+}\right] \cdot 10^{5 \mathrm{~A}(\mathrm{E}-1.507)+8 \mathrm{pH}},\left[\mathrm{MnO}_{4}^{2-}\right]=\left[\mathrm{Mn}^{2+}\right] \cdot 10^{4 \mathrm{~A}(\mathrm{E}-1.743)+8 \mathrm{pH}} \text {, }} \\
& {\left[\mathrm{Mn}^{3+}\right]=\left[\mathrm{Mn}^{2+}\right] \cdot 10^{\mathrm{A}(\mathrm{E}-1.509)},\left[\mathrm{Fe}^{3+}\right]=\left[\mathrm{Fe}^{2+}\right] \cdot 10^{\mathrm{A}(\mathrm{E}-0.771)},\left[\mathrm{H}_{2} \mathrm{CO}_{3}\right]=\left[\mathrm{H}_{2} \mathrm{C}_{2} \mathrm{O}_{4}\right]^{0.5} \cdot 10^{\mathrm{A}(\mathrm{E}+0.396)+\mathrm{pH}} ;}
\end{aligned}
$$

then we have $39=7+31+1$.

Example 13. $\mathrm{V}_{0} \mathrm{~mL}$ of $\mathrm{D}$ containing $\mathrm{KIO}_{3}\left(\mathrm{C}_{0}\right), \mathrm{HCl}\left(\mathrm{C}_{01}\right), \mathrm{H}_{2} \mathrm{SeO}_{3}\left(\mathrm{C}_{02}\right)$ and $\mathrm{HgCl}_{2}\left(\mathrm{C}_{03}\right)$ is titrated with $\mathrm{V}$ $\mathrm{mL}$ of ascorbic acid $\mathrm{C}_{6} \mathrm{H}_{8} \mathrm{O}_{6}(\mathrm{C})$. Within defined volume interval of ascorbic acid solution added, solid iodine, $\mathbf{I}_{2}$ (solubility $\mathrm{s}=1.33 \times 10^{-3} \mathrm{~mol} / \mathrm{L}$ at $20^{\circ} \mathrm{C}$ ) is formed. We have there $\mathrm{P}=46$ species: $\mathrm{H}_{2} \mathrm{O}, \mathrm{H}^{+}, \mathrm{OH}^{-}, \mathrm{e}^{-}, \mathrm{K}^{+}$, $\mathrm{C}_{6} \mathrm{H}_{8} \mathrm{O}_{6}, \mathrm{C}_{6} \mathrm{H}_{7} \mathrm{O}_{6}^{-}, \mathrm{C}_{6} \mathrm{H}_{6} \mathrm{O}_{6}^{2-}, \mathrm{C}_{6} \mathrm{H}_{6} \mathrm{O}_{6}, \mathrm{I}_{2}, \mathrm{I}_{2}, \mathrm{I}^{-}, \mathrm{I}_{3}^{-}, \mathrm{HIO}, \mathrm{IO}^{-}, \mathrm{HIO}_{3}, \mathrm{IO}_{3}^{-}, \mathrm{H}_{5} \mathrm{IO}_{6}, \mathrm{H}_{4} \mathrm{IO}_{6}^{-}, \mathrm{H}_{3} \mathrm{IO}_{6}^{2-}, \mathrm{Cl}_{2}, \mathrm{Cl}^{-}$, $\mathrm{HClO}, \mathrm{ClO}^{-}, \mathrm{HClO}_{2}, \mathrm{ClO}_{2}^{-}, \mathrm{ClO}_{2}, \mathrm{ClO}_{3}^{-}, \mathrm{ClO}_{4}^{-}, \mathrm{I}_{2} \mathrm{Cl}^{-}, \mathrm{ICl}, \mathrm{ICl}_{2}^{-}, \mathrm{H}_{2} \mathrm{SeO}_{3}, \mathrm{HSeO}_{3}^{-}, \mathrm{SeO}_{3}^{2-}, \mathrm{Hg}^{2+}, \mathrm{HgOH}^{+}$, $\mathrm{Hg}(\mathrm{OH})_{2}, \mathrm{HgCl}^{+}, \mathrm{HgCl}_{2}, \mathrm{HgCl}_{3}^{-}, \mathrm{HgCl}_{4}^{2-}, \mathrm{HgI}^{+}, \mathrm{HgI}_{2}, \mathrm{HgI}_{3}^{-}, \mathrm{HgI}_{4}^{2-}$, involved in Q $=8$ balances:

$$
\begin{aligned}
& \left(\mathrm{Z}_{1}+1\right)\left[\mathrm{I}^{-}\right]+\left(3 \mathrm{Z}_{1}+1\right)\left[\mathrm{I}_{3}^{-}\right]+2 \mathrm{Z}_{1}\left(\left[\mathrm{I}_{2}\right]+\alpha \times\left[\mathrm{I}_{2}\right]\right)+\left(\mathrm{Z}_{1}-1\right)\left([\mathrm{HIO}]+\left[\mathrm{IO}^{-}\right]\right)+\left(\mathrm{Z}_{1}-5\right)\left(\left[\mathrm{HIO}_{3}\right]+\left[\mathrm{IO}_{3}^{-}\right]\right) \\
& +\left(\mathrm{Z}_{1}-7\right)\left(\left[\mathrm{H}_{5} \mathrm{IO}_{6}\right]+\left[\mathrm{H}_{4} \mathrm{IO}_{6}^{-}\right]+\left[\mathrm{H}_{3} \mathrm{IO}_{6}^{2-}\right]\right)+\left(\mathrm{Z}_{1}+1\right)\left[\mathrm{HgI}^{+}\right]+2\left(\mathrm{Z}_{1}+1\right)\left[\mathrm{HgI}_{2}\right]+3\left(\mathrm{Z}_{1}+1\right)\left[\mathrm{HgI}_{3}^{-}\right] \\
& +4\left(\mathrm{Z}_{1}+1\right)\left[\mathrm{HgI}_{4}^{2-}\right]+\mathrm{Z}_{3}\left(\left[\mathrm{C}_{6} \mathrm{H}_{8} \mathrm{O}_{6}\right]+\left[\mathrm{C}_{6} \mathrm{H}_{7} \mathrm{O}_{6}^{-}\right]+\left[\mathrm{C}_{6} \mathrm{H}_{6} \mathrm{O}_{6}^{2-}\right]\right)+\left(\mathrm{Z}_{3}-2\right)\left[\mathrm{C}_{6} \mathrm{H}_{6} \mathrm{O}_{6}\right]+\left(\mathrm{Z}_{4}+1\right)\left[\mathrm{Cl}^{-}\right] \\
& +2 \mathrm{Z}_{4}\left[\mathrm{Cl}_{2}\right]+\left(\mathrm{Z}_{4}-1\right)\left([\mathrm{HClO}]+\left[\mathrm{ClO}^{-}\right]\right)+\left(\mathrm{Z}_{4}-3\right)\left(\left[\mathrm{HClO}_{2}\right]+\left[\mathrm{ClO}_{2}^{-}\right]\right)+\left(\mathrm{Z}_{4}-4\right)\left[\mathrm{ClO}_{2}\right] \\
& +\left(\mathrm{Z}_{4}-5\right)\left[\mathrm{ClO}_{3}^{-}\right]+\left(\mathrm{Z}_{4}-7\right)\left[\mathrm{ClO}_{4}^{-}\right]+\left(\mathrm{Z}_{1}+\mathrm{Z}_{4}\right)[\mathrm{ICl}]+\left(\mathrm{Z}_{1}+2\left(\mathrm{Z}_{4}+1\right)\right)\left[\mathrm{ICl}_{2}^{-}\right]+\left(2 \mathrm{Z}_{1}+\mathrm{Z}_{4}+1\right)\left[\mathrm{I}_{2} \mathrm{Cl}^{-}\right] \\
& +\left(\mathrm{Z}_{4}+1\right)\left[\mathrm{HgCl}^{+}\right]+2\left(\mathrm{Z}_{4}+1\right)\left[\mathrm{HgCl}_{2}\right]+3\left(\mathrm{Z}_{4}+1\right)\left[\mathrm{HgCl}_{3}^{-}\right]+4\left(\mathrm{Z}_{4}+1\right)\left[\mathrm{HgCl}_{4}^{2-}\right] \\
& =\left(\left(\mathrm{Z}_{1}-5\right) \mathrm{C}_{0} \mathrm{~V}_{0}+\mathrm{Z}_{3} \mathrm{CV}+\left(\mathrm{Z}_{4}+1\right) \mathrm{C}_{\mathrm{a}} \mathrm{V}_{0}\right) /\left(\mathrm{V}_{0}+\mathrm{V}\right)
\end{aligned}
$$




$$
\begin{aligned}
& {\left[\mathrm{H}^{+}\right]-\left[\mathrm{OH}^{-}\right]-\left[\mathrm{I}^{-}\right]-\left[\mathrm{I}_{3}^{-}\right]-\left[\mathrm{IO}^{-}\right]-\left[\mathrm{IO}_{3}^{-}\right]-\left[\mathrm{H}_{4} \mathrm{IO}_{6}^{-}\right]-2\left[\mathrm{H}_{3} \mathrm{IO}_{6}^{2-}\right]-\left[\mathrm{Cl}^{-}\right]} \\
& -\left[\mathrm{ClO}^{-}\right]-\left[\mathrm{ClO}_{2}^{-}\right]-\left[\mathrm{ClO}_{3}^{-}\right]-\left[\mathrm{ClO}_{4}^{-}\right]-\left[\mathrm{ICl}_{2}^{-}\right]-\left[\mathrm{I}_{2} \mathrm{Cl}^{-}\right]+2\left[\mathrm{Hg}^{2+}\right] \\
& +\left[\mathrm{HgOH}^{+}\right]+\left[\mathrm{HgCl}^{+}\right]-\left[\mathrm{HgCl}_{3}^{-}\right]-2\left[\mathrm{HgCl}_{4}^{2-}\right]+\left[\mathrm{HgI}^{+}\right]-\left[\mathrm{HgI}_{3}^{-}\right] \\
& -2\left[\mathrm{HgI}_{4}^{2-}\right]-\left[\mathrm{C}_{6} \mathrm{H}_{7} \mathrm{O}_{6}^{-}\right]-2\left[\mathrm{C}_{6} \mathrm{H}_{6} \mathrm{O}_{6}^{2-}\right]-\left[\mathrm{HSeO}_{3}^{-}\right]-2\left[\mathrm{SeO}_{3}^{2-}\right]=0 \\
& {\left[\mathrm{I}^{-}\right]+3\left[\mathrm{I}_{3}^{-}\right]+2\left(\left[\mathrm{I}_{2}\right]+\alpha \cdot\left[\mathrm{I}_{2}\right]\right)+[\mathrm{HIO}]+\left[\mathrm{IO}^{-}\right]+\left[\mathrm{HIO}_{3}\right]+\left[\mathrm{IO}_{3}^{-}\right]} \\
& +\left[\mathrm{H}_{5} \mathrm{IO}_{6}\right]+\left[\mathrm{H}_{4} \mathrm{IO}_{6}^{-}\right]+\left[\mathrm{H}_{3} \mathrm{IO}_{6}^{2-}\right]+\left[\mathrm{HgI}^{+}\right]+2\left[\mathrm{HgI}_{2}\right]+3\left[\mathrm{HgI}_{3}^{-}\right] \\
& +4\left[\mathrm{HgI}_{4}^{2-}\right]+[\mathrm{ICl}]+\left[\mathrm{ICl}_{2}^{-}\right]+2\left[\mathrm{I}_{2} \mathrm{Cl}^{-}\right]=\mathrm{C}_{0} \mathrm{~V}_{0} /\left(\mathrm{V}_{0}+\mathrm{V}\right) \\
& {\left[\mathrm{Hg}^{2+}\right]+\left[\mathrm{HgOH}^{+}\right]+\left[\mathrm{Hg}(\mathrm{OH})_{2}\right]+\left[\mathrm{HgCl}^{+}\right]+\left[\mathrm{HgCl}_{2}\right]+\left[\mathrm{HgCl}_{3}^{-}\right]} \\
& +\left[\mathrm{HgCl}_{4}^{2-}\right]+\left[\mathrm{HgI}^{+}\right]+\left[\mathrm{HgI}_{2}\right]+\left[\mathrm{HgI}_{3}^{-}\right]+\left[\mathrm{HgI}_{4}^{2-}\right]=\mathrm{C}_{03} \mathrm{~V}_{0} /\left(\mathrm{V}_{0}+\mathrm{V}\right) \\
& {\left[\mathrm{C}_{6} \mathrm{H}_{8} \mathrm{O}_{6}\right]+\left[\mathrm{C}_{6} \mathrm{H}_{7} \mathrm{O}_{6}^{-}\right]+\left[\mathrm{C}_{6} \mathrm{H}_{6} \mathrm{O}_{6}^{2-}\right]+\left[\mathrm{C}_{6} \mathrm{H}_{6} \mathrm{O}_{6}\right]=\mathrm{CV} /\left(\mathrm{V}_{0}+\mathrm{V}\right)} \\
& {\left[\mathrm{Cl}^{-}\right]+2\left[\mathrm{Cl}_{2}\right]+[\mathrm{HClO}]+\left[\mathrm{ClO}^{-}\right]+\left[\mathrm{HClO}_{2}\right]+\left[\mathrm{ClO}_{2}^{-}\right]+\left[\mathrm{ClO}_{2}\right]} \\
& +\left[\mathrm{ClO}_{3}^{-}\right]+\left[\mathrm{ClO}_{4}^{-}\right]+[\mathrm{ICl}]+2\left[\mathrm{ICl}_{2}^{-}\right]+\left[\mathrm{I}_{2} \mathrm{Cl}^{-}\right]+\left[\mathrm{HgCl}^{+}\right] \\
& +2\left[\mathrm{HgCl}_{2}\right]+3\left[\mathrm{HgCl}_{3}^{-}\right]+4\left[\mathrm{HgCl}_{4}^{2-}\right]=\left(\mathrm{C}_{01}+2 \mathrm{C}_{03}\right) \mathrm{V}_{0} /\left(\mathrm{V}_{0}+\mathrm{V}\right) \\
& {\left[\mathrm{H}_{2} \mathrm{SeO}_{3}\right]+\left[\mathrm{HSeO}_{3}^{-}\right]+\left[\mathrm{SeO}_{3}^{2-}\right]=\mathrm{C}_{02} \mathrm{~V}_{0} /\left(\mathrm{V}_{0}+\mathrm{V}\right)} \\
& {\left[\mathrm{K}^{+}\right]=\mathrm{C}_{0} \mathrm{~V}_{0} /\left(\mathrm{V}_{0}+\mathrm{V}\right)}
\end{aligned}
$$

and $\mathrm{R}=37$ equilibrium constants: $\mathrm{K}_{\mathrm{W}}$ and ones involved in the relations:

$$
\begin{aligned}
& {\left[\mathrm{I}_{2}\right]=\left[\mathrm{I}^{-}\right]^{2} \cdot 10^{2 \mathrm{~A}(\mathrm{E}-0.62)},\left[\mathrm{I}_{3}^{-}\right]=\left[\mathrm{I}^{-}\right]^{3} \cdot 10^{2 \mathrm{~A}(\mathrm{E}-0.545)},} \\
& {\left[\mathrm{IO}^{-}\right]=\left[\mathrm{I}^{-}\right] \cdot 10^{2 \mathrm{~A}(\mathrm{E}-0.49)+2 \mathrm{pH}-28},\left[\mathrm{IO}_{3}^{-}\right]=\left[\mathrm{I}^{-}\right] \cdot 10^{6 \mathrm{~A}(\mathrm{E}-1.08)+6 \mathrm{pH}},} \\
& {[\mathrm{HIO}]=10^{10.6} \cdot\left[\mathrm{H}^{+}\right]\left[\mathrm{IO}^{?}\right],\left[\mathrm{HIO}_{3}\right]=10^{0.79} \cdot\left[\mathrm{H}^{+}\right]\left[\mathrm{IO}_{3}^{-}\right], \quad\left[\mathrm{H}_{5} \mathrm{IO}_{6}\right]=\left[\mathrm{I}^{-}\right] \cdot 10^{8 \mathrm{~A}(\mathrm{E}-1.26)+7 \mathrm{pH}},} \\
& {\left[\mathrm{H}_{4} \mathrm{IO}_{6}^{-}\right]=10^{\mathrm{pH}-3.3} \cdot\left[\mathrm{H}_{5} \mathrm{IO}_{6}\right], \quad\left[\mathrm{H}_{3} \mathrm{IO}_{6}^{2-}\right]=\left[\mathrm{I}^{-}\right] \cdot 10^{8 \mathrm{~A}(\mathrm{E}-0.37)+9 \mathrm{pH}-126}, \quad\left[\mathrm{C}_{6} \mathrm{H}_{6} \mathrm{O}_{6}\right]=\left[\mathrm{C}_{6} \mathrm{H}_{8} \mathrm{O}_{6}\right] \cdot 10^{2 \mathrm{~A}(\mathrm{E}-0.39)+2 \mathrm{pH}} \text {, }} \\
& {\left[\mathrm{C}_{6} \mathrm{H}_{8} \mathrm{O}_{6}\right]=\left[\mathrm{C}_{6} \mathrm{H}_{7} \mathrm{O}_{6}^{-}\right] \cdot 10^{4.21-\mathrm{pH}}, \quad\left[\mathrm{C}_{6} \mathrm{H}_{8} \mathrm{O}_{6}\right]=\left[\mathrm{C}_{6} \mathrm{H}_{6} \mathrm{O}_{6}^{2-}\right] \times 10^{15.78-2 \mathrm{pH}}, \quad\left[\mathrm{I}_{2}\right]=[\mathrm{ICl}]^{2} \cdot 10^{-2 \mathrm{~A}(\mathrm{E}-1.105)+2 \mathrm{pCl}} \text {, }} \\
& {\left[\mathrm{I}_{2} \mathrm{Cl}^{-}\right]=\left[\mathrm{I}_{2}\right] \cdot 10^{0.2-\mathrm{pCl}},\left[\mathrm{ICl}_{2}^{-}\right]=[\mathrm{ICl}] \cdot 10^{2.2-\mathrm{pCl}},\left[\mathrm{H}_{2} \mathrm{SeO}_{3}\right]=10^{2.62} \cdot\left[\mathrm{H}^{+}\right]\left[\mathrm{HSeO}_{3}^{-}\right] \text {, }} \\
& {\left[\mathrm{H}_{2} \mathrm{SeO}_{3}\right]=10^{10.94} \cdot\left[\mathrm{H}^{+}\right]^{2}\left[\mathrm{SeO}_{3}^{2-}\right],\left[\mathrm{HgOH}^{+}\right]=\left[\mathrm{Hg}^{2+}\right] \cdot 10^{\mathrm{pH}-3.7},\left[\mathrm{Hg}(\mathrm{OH})_{2}\right]=\left[\mathrm{Hg}^{2+}\right] \cdot 10^{2 \mathrm{pH}-6.3} \text {, }} \\
& {\left[\mathrm{HgCl}^{+}\right]=\left[\mathrm{Hg}^{2+}\right] \cdot 10^{6.74-\mathrm{pCl}},\left[\mathrm{HgCl}_{2}\right]=\left[\mathrm{Hg}^{2+}\right] \cdot 10^{13.22-2 \mathrm{pCl}} \text {, }} \\
& {\left[\mathrm{HgCl}_{3}^{-}\right]=\left[\mathrm{Hg}^{2+}\right] \cdot 10^{14.07-3 \mathrm{pCl}},\left[\mathrm{HgCl}_{4}^{2-}\right]=\left[\mathrm{Hg}^{2+}\right] \cdot 10^{15.07-4 \mathrm{pCl}},\left[\mathrm{HgI}^{+}\right]=\left[\mathrm{Hg}^{2+}\right] \cdot 10^{12.87-\mathrm{pl}} \text {, }} \\
& {\left[\mathrm{HgI}_{2}\right]=\left[\mathrm{Hg}^{2+}\right] \cdot 10^{23.8-2 \mathrm{pI}},\left[\mathrm{HgI}_{3}^{-}\right]=\left[\mathrm{Hg}^{2+}\right] 10^{27.6-3 \mathrm{pI}},\left[\mathrm{HgI}_{4}^{2-}\right]=\left[\mathrm{Hg}^{2+}\right] 10^{29.6-4 \mathrm{pI}} \text {, }} \\
& {\left[\mathrm{Cl}_{2}\right]=10^{2 \mathrm{~A}(\mathrm{E}-1.359)-2 \mathrm{pCl}},\left[\mathrm{ClO}^{-}\right]=10^{2 \mathrm{~A}(\mathrm{E}-0.88)+2 \mathrm{pH}-\mathrm{pCl}-28},[\mathrm{HClO}]=10^{7.3}\left[\mathrm{H}^{+}\right]\left[\mathrm{ClO}^{-}\right] \text {, }} \\
& {\left[\mathrm{ClO}_{2}^{-}\right]=10^{4 \mathrm{~A}(\mathrm{E}-0.77)+4 \mathrm{pH}-\mathrm{pCl}-56},\left[\mathrm{HClO}_{2}\right]=10^{1.97}\left[\mathrm{H}^{+}\right]\left[\mathrm{ClO}_{2}^{-}\right] \text {, }} \\
& {\left[\mathrm{ClO}_{2}\right]=10^{5 \mathrm{~A}(\mathrm{E}-1.5)+4 \mathrm{pH}-\mathrm{pCl}},\left[\mathrm{ClO}_{3}^{-}\right]=10^{6 \mathrm{~A}(\mathrm{E}-1.45)+6 \mathrm{pH}-\mathrm{pCl}},\left[\mathrm{ClO}_{4}^{-}\right]=10^{8 \mathrm{~A}(\mathrm{E}-1.38)+8 \mathrm{pH}-\mathrm{pCl}}}
\end{aligned}
$$


where: A $=16.92$ (at $298 \mathrm{~K}$ ), $\mathrm{pCl}=-\log \left[\mathrm{Cl}^{-}\right]$, $\mathrm{pI}=-\log \left[\mathrm{I}^{-}\right]$; the solubility s of $\mathbf{I}_{2}$ is the 37th equilibrium constant. Within $\Phi$ range where $\mathbf{I}_{2}$ is the equilibrium solid phase, we have $46=8+37+1$; if not (i.e. $\alpha=0$ in Equations (17) and (18)), we have $45=8+36+1$.

Example 14. $\mathrm{V}$ mL NaOH $(\mathrm{C})+\mathrm{CO}_{2}\left(\mathrm{C}_{1}\right)$ is added into $\mathrm{V}_{0} \mathrm{~mL}$ of $\mathrm{I}_{2}\left(\mathrm{C}_{0}\right)+\mathrm{KI}\left(\mathrm{C}_{01}\right)+\mathrm{CO}_{2}\left(\mathrm{C}_{02}\right)+\mathrm{W}_{(\mathrm{o})} \mathrm{mL}$ of $\mathrm{CCl}_{4}$ [37]. In the liquid-liquid extraction system thus obtained we have: $\mathrm{P}=20\left(\mathrm{H}_{2} \mathrm{O}, \mathrm{H}^{+}, \mathrm{OH}^{-}, \mathrm{e}^{-}, \mathrm{I}^{-}, \mathrm{I}_{3}^{-}, \mathrm{I}_{2}\right.$, $\left.\mathbf{I}_{2(\mathrm{~s})}, \mathrm{I}_{2(\mathrm{o})}, \mathrm{HIO}, \mathrm{IO}^{-}, \mathrm{HIO}_{3}, \mathrm{IO}_{3}^{-}, \mathrm{H}_{5} \mathrm{IO}_{6}, \mathrm{H}_{4} \mathrm{IO}_{6}^{-}, \mathrm{H}_{3} \mathrm{IO}_{6}^{2-}, \mathrm{H}_{2} \mathrm{CO}_{3}, \mathrm{HCO}_{3}^{-}, \mathrm{CO}_{3}^{2-}, \mathrm{CCl}_{4}\right), \mathrm{Q}=5$ (GEB, charge, I, $\left.\mathrm{C}, \mathrm{CCl}_{4}\right), \mathrm{R}=14$; then $20=5+14+1$.

\section{General Remarks}

The equilibrium data on electrolytic systems refer almost exclusively to aqueous solutions. The relevant data for the systems with non-aqueous solvents are fragmentary and relate almost exclusively to acid-base equilibria. In the binary-solvent acid-base systems, the $\mathrm{pK}_{\mathrm{i}}$ values for dissociation constants $\mathrm{K}_{\mathrm{i}}$ are replaced by dissociation parameters, $\mathrm{pK}_{\mathrm{i}}(\mathrm{x})$, as functions of the mole fraction $\mathrm{x}$ of one of the co-solvents in the related mixtures [13] [48] [49]. Therefore, in further remarks we will refer only to aqueous solutions.

Generalizing, any electrolytic non-redox systems is characterized by one charge balance, and one or more concentration balances. The concentration balances are obtained from elemental or core balances, related to elements $\mathrm{Y}_{\mathrm{i}} \neq \mathrm{H}$, O.

Any redox system is characterized by one charge balance, one electron balance, named as generalized electron balance (GEB), and one or more concentration balances for elements/cores $Y_{i} \neq H$, O. The GEB can be formulated according to the Approach I to GEB or according to the Approach II to GEB. The Approach I, named also as a "short" version of GEB, is realized under assumption that the oxidation numbers for all elements of the system considered are known beforehand. The Approach II to GEB is formulated on the basis of linear combination $2 \cdot f(\mathrm{O})-f(\mathrm{H})$ of elemental balances: $f(\mathrm{H})$ for $\mathrm{H}$ and $f(\mathrm{O})$ for $\mathrm{O}$. Any linear combination of $2 \cdot f(O)-f(H)$ with charge and elemental/core balances has full properties of GEB. The GEB is expressed in terms of concentrations of the related species, as charge and concentration balances.

\section{Some Remarks on the Systems Considered}

Equation (1) was checked in on numerous examples, related to electrolytic systems of different degree of complexity, and concluding formula was obtained in deductive manner. The non-redox and redox systems are distinguished on the basis of properties of the equation $2 \cdot f(O)-f(H)$ [5] [11]. The electrolytic systems are considered as closed systems, composed only of condensed (liquid, solid) phases. Any process in closed system can be realied under isothermal conditions; constant temperature (next to ionic strength) is one of prerequisities securing constancy of the equilibrium constants.

In the examples, well, moderately, or sparingly soluble species are involved in batch or dynamic (titration) systems. An action of some components on the phase composition is also considered. The effect of an excess of the corresponding regent on phase composition is also taken into account. Electron is considered among the species in redox systems. GEB is considered as a one of the balances needed for mathematical description of redox systems, as fully compatible with charge and concentration balances related to the system in question.

\section{Final Comments}

Equation (1) deduced in this paper is in close relevance to GATES [1]-[18] where one can use in calculations all attainable knowledge contained in thermodynamic equilibrium constants, regarding mono- and/or multiphase systems. It has been shown that the proposed relation is adequate for systems of different and varying complexity. In redox systems, electrons are considered as species. In particular, Equation (1) is valid for two- and threephase redox systems. The validity of Equation (1) has been demonstrated in static and dynamic systems. The quasistatic course of the appropriate changes occurring under isothermal conditions is illustrated graphically. On this basis, the reactions involving the dominant components in these systems were formulated.

Omission/introduction of a species in the related balance(s) is involved with omission/introduction of the related equilibrium constant. Therefore, the Equation (1) is valid after such operations, $(\mathrm{P} \pm \mathrm{m})=\mathrm{Q}+(\mathrm{R} \pm \mathrm{m})+1$ $\Leftrightarrow \mathrm{P}=\mathrm{Q}+\mathrm{R}+1$. 


\section{References}

[1] Michałowski, T. (2011) Application of GATES and MATLAB for Resolution of Equilibrium, Metastable and NonEquilibrium Electrolytic Systems. In: Michałowski, T., Ed., Applications of MATLAB in Science and Engineering, InTech-Open Access Publisher in the Fields of Science, Technology and Medicine, Rijeka, 1-34.

http://www.intechopen.com/books/show/title/applications-of-matlab-in-science-and-engineering http://dx.doi.org/10.5772/19538

[2] Michałowski, T. (2010) The Generalized Approach to Electrolytic Systems: I. Physicochemical and Analytical Implications. Critical Reviews in Analytical Chemistry, 40, 2-16. http://dx.doi.org/10.1080/10408340903001292

[3] Michałowski, T., Pietrzyk, A., Ponikvar-Svet, M. and Rymanowski, M. (2010) The Generalized Approach to Electrolytic Systems: II. The Generalized Equivalent Mass (GEM) Concept, Critical Reviews in Analytical Chemistry, 40, 1729. http://dx.doi.org/10.1080/10408340903001342

[4] Michałowski, T., Toporek, M., Michałowska-Kaczmarczyk, A.M. and Asuero, A.G. (2013) New Trends in Studies on Electrolytic Redox Systems. Electrochimica Acta, 109, 519-531. http://dx.doi.org/10.1016/j.electacta.2013.07.125

[5] Michałowski, T., Michałowska-Kaczmarczyk, A.M. and Toporek, M. (2013) Formulation of General Criterion Distinguishing between Non-Redox and Redox Systems. Electrochimica Acta, 112, 199-211. http://dx.doi.org/10.1016/j.electacta.2013.08.153

[6] Michałowska-Kaczmarczyk, A.M., Toporek, M. and Michałowski, T. (2015) Speciation Diagrams in Dynamic Iodide + Dichromate System. Electrochimica Acta, 155, 217-227. http://dx.doi.org/10.1016/j.electacta.2015.01.003

[7] Toporek, M., Michałowska-Kaczmarczyk, A.M. and Michałowski, T. (2015) Symproportionation versus Disproportionation in Bromine Redox Systems. Electrochimica Acta, 171, 176-187. http://dx.doi.org/10.1016/j.electacta.2015.05.012

[8] Michałowska-Kaczmarczyk, A.M., Asuero, A.G., Toporek, M. and Michałowski, T. (2015) "Why Not Stoichiometry” versus “Stoichiometry-Why Not?” Part II. GATES in Context with Redox Systems. Critical Reviews in Analytical Chemistry, 45, 240-268. http://dx.doi.org/10.1080/10408347.2014.937853

[9] Michałowska-Kaczmarczyk, A.M., Michałowski, T., Toporek, M. and Asuero, A.G. (2015) "Why Not Stoichiometry" versus “Stoichiometry—Why Not?” Part III, Extension of GATES/GEB on Complex Dynamic Redox Systems. Critical Reviews in Analytical Chemistry, 45, 348-366.

http://www.tandfonline.com/doi/abs/10.1080/10408347.2014.953673

http://dx.doi.org/10.1080/10408347.2014.953673

[10] Michałowski, T., Ponikvar-Svet, M., Asuero, A.G. and Kupiec, K. (2012) Thermodynamic and Kinetic Effects Involved in the $\mathrm{pH}$ Titration of As(III) with Iodine in a Buffered Malonate System. Journal of Solution Chemistry, 41, 436-446. http://dx.doi.org/10.1007/s10953-012-9815-6

[11] Michałowska-Kaczmarczyk, A.M. and Michałowski, T. (2013) Comparative Balancing of Non-Redox and Redox Electrolytic Systems and Its Consequences. American Journal of Analytical Chemistry, 4, 46-53. http://file.scirp.org/Html/38569.html http://dx.doi.org/10.4236/ajac.2013.410A1006

[12] Michałowska-Kaczmarczyk, A.M. and Michałowski, T. (2014) Compact Formulation of Redox Systems According to GATES/GEB Principles. Journal of Analytical Sciences, Methods and Instrumentation, 4, 39-45. http://www.scirp.org/journal/PaperInformation.aspx?PaperID=46335

[13] Michałowski, T., Pilarski, B., Asuero, A.G. and Michałowska-Kaczmarczyk, A.M. (2014) Chapter 9.4: Modeling of Acid-Base Properties in Binary-Solvent Systems. In: Wypych, G., Ed., Handbook of Solvents, 2nd Edition, Vol. 1, ChemTec Publishing, Toronto, 623-648. http://www.sciencedirect.com/science/book/9781895198645

[14] Michałowska-Kaczmarczyk, A.M. and Michałowski, T. (2014) Generalized Electron Balance for Dynamic Redox Systems in Mixed-Solvent Media. Journal of Analytical Sciences, Methods and Instrumentation, 4, 102-109. http://www.scirp.org/Journal/PaperInformation.aspx?PaperID=52018\#.VH1N5Gfpt74

[15] Michałowska-Kaczmarczyk, A.M., Rymanowski, M., Asuero, A.G., Toporek, M. and Michałowski, T. (2014) Formulation of Titration Curves for Some Redox Systems. American Journal of Analytical Chemistry, 5, 861-878. http://www.scirp.org/journal/PaperInformation.aspx?paperID=50016\#.VGOZ92dvHFw http://dx.doi.org/10.4236/ajac.2014.513095

[16] Toporek, M., Michałowska-Kaczmarczyk, A.M. and Michałowski, T. (2014) Disproportionation Reactions of HIO and NaIO in Static and Dynamic Systems. American Journal of Analytical Chemistry, 5, 1046-1056. http://www.scirp.org/journal/PaperInformation.aspx?PaperID=51637\#.VHXKcWfpt74 http://dx.doi.org/10.4236/ajac.2014.515111

[17] Michałowska-Kaczmarczyk, A.M. and Michałowski, T. (2014) GATES as the Unique Tool for Simulation of Electrolytic Redox and Non-Redox Systems. Journal of Analytical \& Bioanalytical Techniques, 5, 204. 
http://omicsonline.org/open-access/gates-as-the-unique-tool-for-simulation-of-electrolytic-redox-and-non-redox-syste ms-2155-9872.1000204.pdf http://dx.doi.org/10.4172/2155-9872.1000204

[18] Michałowski, T. (2014) Generalized Electron Balance (GEB) as a Law of Preservation for Electrolytic Redox Systems. http://myprogram14.ise-online.org/infiles/pdf/ise141331.pdf

[19] Inczédy, J. (1976) Analytical Applications of Complex Equilibria. Ellis Horwood, Chichester.

[20] Nikolsky, B.P. (1964) Guide-Book for Chemist. Vol. 3, Khimia, Moscow. (In Russian)

[21] Asuero, A.G. and Michałowski, T. (2011) Comprehensive Formulation of Titration Curves Referred to Complex Acid-Base Systems and Its Analytical Implications. Critical Reviews in Analytical Chemistry, 41, 151-187. http://dx.doi.org/10.1080/10408347.2011.559440

[22] http://en.wikipedia.org/wiki/Solubility_equilibrium

[23] Sillén, L.G. and Martell, A.E. (1971) Stability Constants of Metal-Ion Complexes. The Chemical Society, London 1964, Supplement No. 1.

[24] Michałowska-Kaczmarczyk, A.M. and Michałowski, T. (2015) Solubility Product Challenge. Analytical and Bioanalytical Chemistry, 407, 1789-1791. http://dx.doi.org/10.1007/s00216-014-8407-2

[25] Michałowska-Kaczmarczyk, A.M. and Michałowski, T. (2015) Solution to Solubility Product Challenge. Analytical and Bioanalytical Chemistry, 407, 4877-4878. http://dx.doi.org/10.1007/s00216-015-8713-3

[26] Michałowska-Kaczmarczyk, A.M. and Michałowski, T. (2014) Calculation of Solubility of Oxyquinolinates. Journal of Analytical Sciences, Methods and Instrumentation, 4, 71-79.

[27] Michałowska-Kaczmarczyk, A.M., Asuero, A.G. and Michałowski, T. (2015) "Why Not Stoichiometry” versus "Stoichiometry-Why Not?” Part I: General Context. Critical Reviews in Analytical Chemistry, 45, 166-188. http://www.ncbi.nlm.nih.gov/pubmed/25558777 http://dx.doi.org/10.1080/10408347.2014.937852

[28] Freiser, H. (1992) Concepts \& Calculations in Analytical Chemistry: A Spreadsheet Approach. CRC Press, Inc., Boca Raton.

[29] Michałowski, T. (1992) Solving Acid-Base Equilibria: Some Concerns. Journal of Chemical Education, 69, 858-859. http://dx.doi.org/10.1021/ed069p858

[30] Michałowski, T. and Pietrzyk, A. (2006) The Generalized Electron Balance Concept. Derivation Based on Elementary Rules of the Matter Conservation. In: Zuba, D. and Parczewski, A., Eds., Chemometrics: Methods and Applications, Kraków, 415-422.

[31] Michałowski, T. (2007) Complementarity of Physical and Chemical Laws of Preservation in Aspect of Electrolytic Systems. Wiadomości Chemiczne, 61, 625-640. (In Polish) http://yadda.icm.edu.pl/yadda/element/bwmeta1.element.baztech-article-BUS5-0004-0047

[32] Marx, D., Tuckerman, M.E., Hutter, J. and Parrinello, M. (1999) The Nature of the Hydrated Excess Proton in Water. Nature, 397, 601-604. http://dx.doi.org/10.1038/17579

[33] Silverstein, T.P. (2014) The Aqueous Proton Is Hydrated by More Than One Water Molecule: Is the Hydronium Ion a Useful Conceit? Journal of Chemical Education, 91, 608-610. http://dx.doi.org/10.1021/ed400559t

[34] Michałowska-Kaczmarczyk, A.M. and Michałowski, T. (2014) Linear Dependence of Balances for Non-Redox Electrolytic Systems. American Journal of Analytical Chemistry, 5, 1285-1289. http://www.scirp.org/journal/PaperInformation.aspx?paperID=52615 http://dx.doi.org/10.4236/ajac.2014.517134

[35] Michalowski, T. (1994) Calculation of pH and Potential E for Bromine Aqueous Solutions. Journal of Chemical Education, 71, 560-562. http://dx.doi.org/10.1021/ed071p560

[36] Michałowski, T. and Lesiak, A. (1994) Acid-Base Titration Curves in Disproportionating Redox Systems. Journal of Chemical Education, 71, 632-636. http://dx.doi.org/10.1021/ed071p632

[37] Michałowski, T. (2001) Calculations in Analytical Chemistry with Elements of Computer Programming. Cracow, PK. (In Polish) http://suw.biblos.pk.edu.pl/resourceDetails\&rId=3974

[38] Michałowski, T., Baterowicz, A., Madej, A. and Kochana, J. (2001) An Extended Gran Method and Its Applicability for Simultaneous Determination of Fe(II) and Fe(III). Analytica Chimica Acta, 442, 287-293. http://dx.doi.org/10.1016/S0003-2670(01)01172-2

[39] Michałowski, T., Rymanowski, M. and Pietrzyk, A. (2005) Nontypical Brönsted’s Acids and Bases. Journal of Chemical Education, 82, 470-472. http://dx.doi.org/10.1021/ed082p470

[40] Michałowski, T., Toporek, M. and Rymanowski, M. (2005) Overview on the Gran and Other Linearization Methods 
Applied in Titrimetric Analyses. Talanta, 65, 1241-1253. http://dx.doi.org/10.1016/j.talanta.2004.08.053

[41] Michałowski, T., Kupiec, K. and Rymanowski, M. (2008) Numerical Analysis of the Gran Methods: A Comparative Study. Analytica Chimica Acta, 606, 172-183. http://dx.doi.org/10.1016/j.aca.2007.11.020

[42] Ponikvar, M., Michałowski, T., Kupiec, K., Wybraniec, S. and Rymanowski, M. (2008) Experimental Verification of the Modified Gran Methods Applicable to Redox Systems. Analytica Chimica Acta, 628, 181-189. http://dx.doi.org/10.1016/j.aca.2008.09.012

[43] Burgot, J.L. (2012) Ionic Equilibria in Analytical Chemistry. Springer, New York. http://dx.doi.org/10.1007/978-1-4419-8382-4

[44] Sudha, P.D.C. (2012) Pharmaceutical Analysis. Pearson Education, New Delhi.

[45] Kotrlý, S. and Šůcha, L. (1985) Handbook of Chemical Equlibria in Analytical Chemistry. Ellis Horwood Limited, Halsted Press, John Wiley \& Sons, New York.

[46] Michałowski, T. and Pietrzyk, A. (2006) A Thermodynamic Study of Struvite + Water System. Talanta, 68, 594-601. http://dx.doi.org/10.1016/j.talanta.2005.04.052

[47] Michałowski, T., Asuero, A.G., Ponikvar-Svet, M., Toporek, M., Pietrzyk, A. and Rymanowski, M. (2012) Principles of Computer Programming Applied to Simulated $\mathrm{pH}$-Static Titration of Cyanide According to a Modified Liebig-Denigès Method. Journal of Solution Chemistry, 41, 1224-1239.

[48] Pilarski, B., Dobkowska, A., Foks, H. and Michałowski, T. (2010) Modeling of Acid-Base Equilibria in Binary-Solvent Systems: A Comparative Study. Talanta, 80, 1073-1080. http://dx.doi.org/10.1016/j.talanta.2009.07.033

[49] Michałowski, T., Pilarski, B., Dobkowska, A. and Młodzianowski, J. (2010) Mathematical Modeling and Physicochemical Studies on Acid-Base Equilibria in Binary-Solvent Systems. Wiadomości Chemiczne, 54, 124-154. 